\section{F.-H. CORNET}

Institut de Physique

du Globe de Paris

4. place Jussieu

75252 Paris Cedex 05 cornet@ipgp.jussieu.fr

\title{
L'étude in situ du rôle des fluides en mécanique crustale
}

L'augmentation de pression interstitielle dans un massif rocheux, lorsqu'elle est assez élevée, induit une activité microsismique. L'analyse de cette microsismicité peut permettre de précíser le champ de contrainte dans le massif mais aussi le champ de pression interstitielle. Toutefois les fractures significatives du point de vue hydraulique étant fréquemment sources d'hétérogénéité de contrainte, Yanalyse de la microsismicité en termes de champ de contrainte et de pression doit toujours être accompagnée d'observations indépendantes: mesures hydrauliques en forage, données d'ovalisation de forage, directions d'anisotropie sismique. L'intégration des données indépendantes et des mécanismes au foyer de la microsismicité permet d'identifier les zones hétérogènes. Ces principes ont été appliqués sur trois sites expérimentaux. Sur le site granitique du Mayet-de-Montagne dans des forages de $800 \mathrm{~m}$ de profondeur la méthode a été testée et validée. Sur le site géothermique de Soultz-sous-Forêts dans un forage de $3500 \mathrm{~m}$ de profondeur, cette méthode permet de définir le domaine de pression interstitielle pour lequel la migration du front de sismicité dépend de la perméabilité du milieu et le seuil de pression au-delà duquel interviennent des processus de rupture majeurs dans le massif. Le troisième site concerne un forage de $1800 \mathrm{~m}$ de profondeur recoupant la faille philippine (plus de $1200 \mathrm{~km}$ de long) qui flue à la vitesse moyenne de $3 \mathrm{~cm} / \mathrm{an}$. Les résultats suggèrent que la faille est très peu perméable et qu'elle est perpendiculaire à la contrainte principale minimale. Ces méthodes d'observation sont actuellement mises en œuvre pour étudier in situ le rôle de la percolation de fluides profond dans le mécanisme d'ouverture du rift de Corinthe.

Mots-clés : mícrosismicité induite, mécanismes au foyer, contrainte, pression interstitielle.

\section{In situ study of the role of fluids in crustal mechanics}

When the interstitial pressure in a rock mass is increased significantly, it induces a microseismic activity which may help to determine the stress field in the rock mass as well as the local pore pressure. However, because permeable fractures are often associated with local stress heterogeneity, the sole focal mechanisms of induced seismicity may yield biased results. Hence they must be associated with additional independent data for a satisfactory stress field characterization : hydraulic tests in

boreholes, borehole breakouts orientations, direction of anisotropy of shear wave velocity field. These principles have been applied at three different sites. At the granitic site of le Mayet-de-Montagne (central France), the method has been tested and validated in two boreholes $800 \mathrm{~m}$ deep, $100 \mathrm{~m}$ apart. At the Geothermal site of Soultz-sous-Forêts, the method provides means to determine the interstitial pressure domain for which the growth of seismic cloud is a good indicator of the large scale rock mass permeability. It is shown that when the pore pressure reaches a critical value, it induces large scale failure processes so that the microseismic cloud migration is no more indicative of large scale permeability. At Tongonan, in the Philippine, an $1800 \mathrm{~m}$ deep well intersects the $1200 \mathrm{~km}$ long Philippine fault in a creeping segment of the fault $(3 \mathrm{~cm} /$ year). Induced seismicity associated to a large scale fluid injection shows that the fault is impervious and perpendicular to the minimum principal stress direction in this area. This observation technique is presently being applied in the Corinth Gulf (Greece) so as to document by in situ observation the role of fluids on the opening process of this rift.

Key words : induced microseismicity, focal mechanism, stress, pore pressure. 


\section{Introduction}

Un des problèmes classiques de la géotechnique est l'extrapolation des résultats obtenus au laboratoire aux conditions réelles du terrain. Il est ainsi bien connu que les modules d'élasticité mesurés sur de petits échantillons ne correspondent pas aux modules à prendre en compte pour évaluer les déformations observées lors des sollicitations réelles. Il est également bien connu que les mesures dynamiques de ces mèmes modules, qui elles peuvent être réalisées sur le terrain à la bonne échelle, ne fournissent pas de résultats satisfaisants pour la prévision des déformations quasi statiques. Ces difficultés proviennent tant d'une mauvaise appréhension de la rhéologie du matériau continu équivalent considéré que d'un manque de connaissance quant à la morphologie et aux propriétés mécaniques des discontinuités majeures du terrain.

En l'absence de réponse satisfaisante à cette question, l'ingénieur géotechnicien a développé des règles plus ou moins empiriques pour produire les valeurs requises par ses analyses. Il s'assure de la fiabilité de ses résultats en réalisant quelques observations à l'échelle 1. De plus, ses observations de terrain, en lui fournissant les données à la bonne échelle, font avancer sa compréhension des mécanismes impliqués et lui permettent de proposer des lois reflétant mieux la physique réelle des phénomènes mis en jeu.

De même, il est apparu nécessaire de mener in situ des observations à l'échelle 1 pour faire progresser notre compréhension des divers phénomènes qui gouvernent la mécanique de la croûte terrestre. Ainsi par exemple, les mesures dans le forage de Cajon Pass, au voisinage de la faille San Andreas en Californie, ont bien mis en évidence l'absence d'anomalie thermique locale ainsi que l'absence de contraintes de cisaillement dans le plan de faille (Lachenbruch et Sass, 1992; Zoback et Healy, 1992). Ces résultats ont, de facto, démontré les limites de notre compréhension des lois de frottement à prendre en compte pour cette faille transformante, site d'un décrochement de plus de $300 \mathrm{~km}$ entre les plaques Pacifique et Nord-Amérique. Il est aujourd'hui reconnu qu'une des lacunes majeures de la géomécanique reste la formalisation des lois de comportement des failles et des différents couplages (hydro-thermo-chimio-mécanique) associés aux circulations de fluides qui y interviennent.

Dans cet article, nous montrons comment des analyses de sismicité induite, observées à l'occasion d'études de réservoirs géothermiques, ont fourni les données requises pour déterminer le champ de contrainte et de pression interstitielle locale. Ces observations ont permis d'étudier in situ les processus de rupture d'un massif granitique dans l'Est de la France et certains aspects du comportement mécanique d'une faille transformante majeure aux Philippines.

\section{2}

\section{Microsismicité et piézométrie in situ: les résultats du Mayet-de-Montagne}

\section{1}

\section{Contexte expérimental}

Les données utilisées ici ont été obtenues dans le cadre d'une étude sur les possibilités de développer une méthode d'exploitation de la chaleur des roches chaudes profondes peu perméables. Il s'agissait de stimuler par méthode hydraulique le réseau de fractures naturelles d'un massif granitique pour y développer un échangeur thermique efficace. La procédure d'injection devait permettre d'éviter de développer des fractures hydrauliques vraies, c'est-à-dire orientées perpendiculairement à la contrainte principale minimale dans le milieu et qui se seraient refermées à l'issue des essais. Nous souhaitions plutôt induire des cisaillements le long de plans de fracture préexistants de façon à en augmenter la conductivité hydraulique du fait de la dilatance associée (Cornet, 1989).

Deux forages d'environ $800 \mathrm{~m}$ de profondeur et distants l'un de l'autre d'une centaine de mètres, permettaient d'injecter, dans le premier, l'eau qui, après circulation dans le réseau de fractures stimulées, devait ressortir par le second pour être recyclée après refroidissement. La cartographie de la sismicité induite associée à des débitmétries en forage devait permettre de caractériser l'écoulement dans le réseau de fracture.

Le site choísi, au Mayet-de-Montagne, à $25 \mathrm{~km}$ au sud-est de Vichy, ètait implanté dans un massif granitique affleurant, ce qui facilitait tant l'étude structurale du massif que l'implantation des 15 capteurs sismiques à trois composantes utilisés pour localiser les événements microsismiques induits (Talebi et Cornet, 1987).

Préalablement aux essais, le champ de fractures naturelles recoupées par les forages avait été identifié par une méthode d'imagerie électrique, nouvelle à l'époque (Mosnier, 1982). Le champ de contrainte avait été déterminé par la méthode HTPF, spécialement développée pour ces besoins (Cornet et Valette, 1984). La méthode HTPF implique la détermination par essais hydrauliques de la contrainte normale $\sigma$ supportée par divers plans de normale n d'orientation connue et variée:

$$
\sigma_{\mathrm{n}}=\Omega(\mathrm{x}) \mathrm{n} \cdot \mathrm{n}
$$

L'objectif est de déterminer, pour $\mathrm{N}$ mesures HTPF $(\mathrm{N} \geq 6$ ), le tenseur complet $\sigma(x)$. Lorsque les points de mesure $x$ (intersection des fractures préexistantes avec le forage) sont distants de plus d'une trentaine de mètres les uns des autres, on suppose que les variations de contrainte restent faibles pour être approximées par un développement limité du premier ordre:

$\underline{\sigma}(\mathrm{x})=\underline{\sigma}\left(\mathrm{x}_{c}\right)+\left(\mathrm{x}_{1}-\mathrm{x}_{1 c}\right) \underline{\alpha}_{1}+\left(\mathrm{x}_{2}-\mathrm{x}_{2 c}\right) \underline{\alpha}_{2}+\left(\mathrm{x}_{3}-\mathrm{x}_{3 c}\right) \underline{\alpha}_{3}$ où $\sigma(x)$ est le tenseur de contrainte en un point $x$ de référence et oủ $\underline{\alpha}_{1}, \underline{\alpha}_{2}$ et $\underline{\alpha}_{3}$ sont les gradients de contrainte le long des trois axes du repère choisi. Il est à noter que l'équation (2) implique 24 paramètres, dans le cas général. Par ailleurs, cette équation doit satisfaire les conditions d'équilibre du massif, ce qui introduit trois équations mais rajoute une inconnue (la masse volumique). Souvent cependant, la masse volumique des terrains est connue, de sorte qu'en fait, cette approximation du champ de contrainte implique 21 paramètres. Lorsque les variations horizontales de contrainte sont négligeables $\left(\underline{\alpha}_{1}=\underline{\alpha}_{2}=\underline{0}\right)$ on démontre que la direction verticale est principale pour le gradient vertical $\underline{\alpha}_{3}$ (McGarr, 1982), de sorte que l'équation (2) n'implique que 10 paramètres ( 9 si la masse volumique est connue). Dans certains cas, il est possible de n'en considérer que 6 , si le massif est homogène jusqu'à la surface du sol, et 5 si l'on néglige les rotations de directions principales avec la profondeur.

Après de nombreux tâtonnements, les opérations de stimulation se sont achevées par une injection d'envi- 
ron $16500 \mathrm{~m}^{3}$ d'eau à des débits croissants par pallier, de quelques jours chacun, et variant de $8,5 \mathrm{l} / \mathrm{s}$ à $21,2 \mathrm{l} / \mathrm{s}$, pour des pressions croissant régulièrement (Fig. 1).

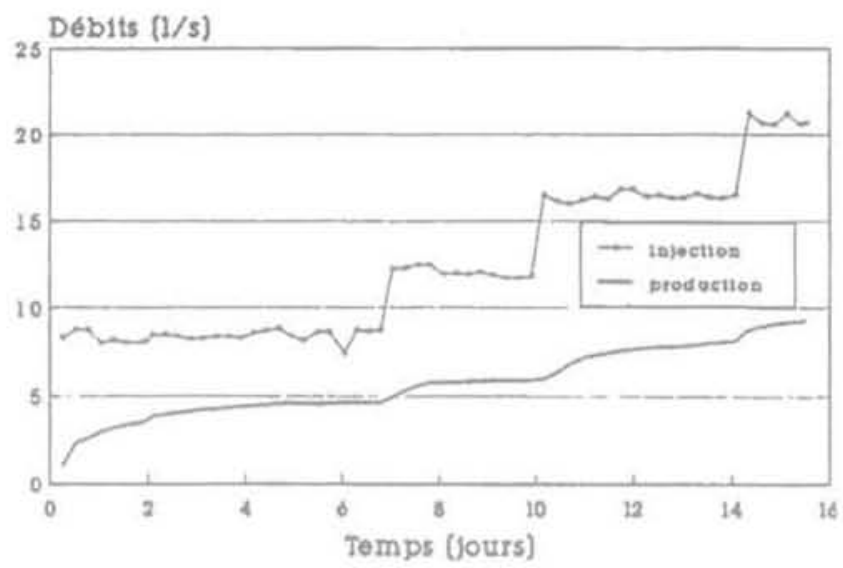

FG.1 Débits d'injection, et de production, à la fin des opérations de stimulation, au Mayet-de-Montagne.

Puis l'injection s'est poursuivie à divers débits décroissants pour caractériser l'impédance hydraulique du système. La relation débit d'injection - pression d'injection est indiquée sur la figure 2. Il est intéressant de comparer ces résultats à ceux obtenus à Soultz, dans des conditions similaires de débit, mais pour lesquels on observe une stabilisation de la pression (Fig. 6).
Dans le cas du Mayet-de-Montagne, la pression dans le forage en fin d'essai, au niveau de l'injection, était notoirement supérieure à la contrainte principale minimale dans le milieu (Fig. 4), ce qui démontre l'ímportance de l'histoire de la montée en pression pour prévoir la réponse du système (absence de fracturation hydraulique dans ce cas particulier).

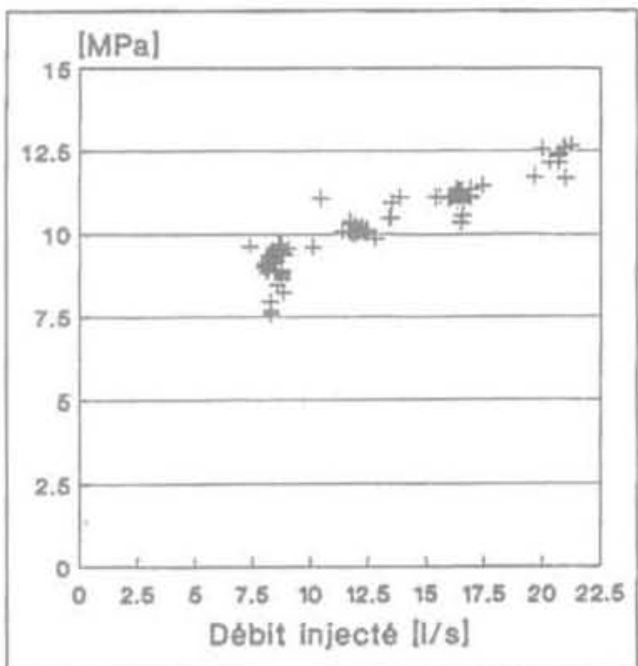

FG. 9 Courbes débit-pression pour la fin des essais de stimulation. Noter l'augmentation régulière de pression.

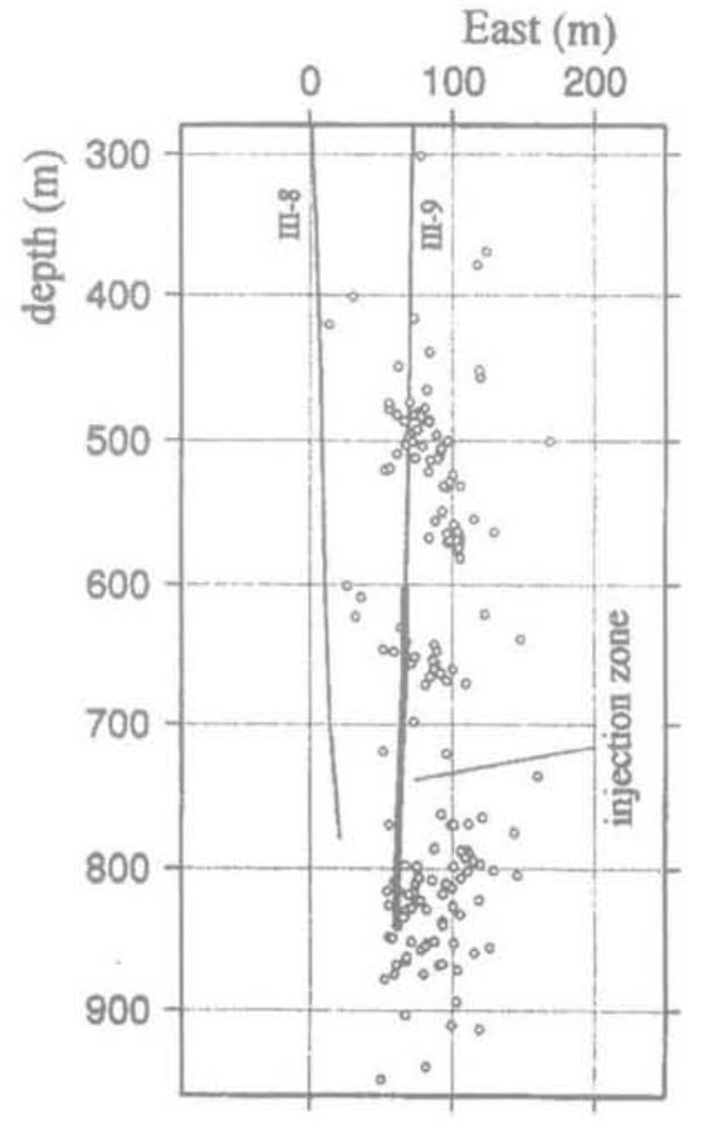

B)

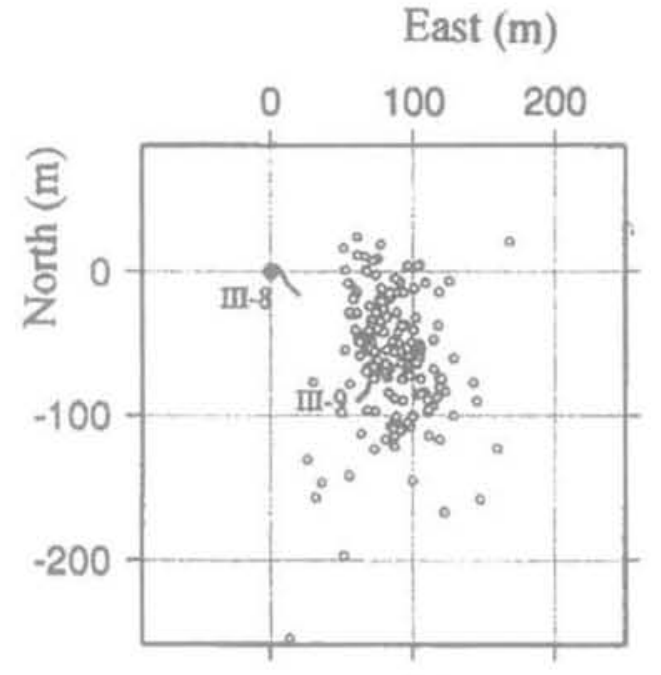

A)

FIG.3 Localisation des événements microsismiques induits lors des divers essais de stimulation: A) projection dans le plan horizontal; B) projection sur un plan vertical est-ouest. 
Quelque 200 événements microsismiques ont été observés (on n'a considéré ici que les sources sismiques assimilables à des doubles couples ${ }^{(1)}$, à l'exclusion des événements dits longue période) (Fig. 3). Pour seulement 87 mécanismes au foyer les deux plans nodaux ont pu être bien contraints à partir des données de polarité des ondes $\mathrm{P}$. Ces mécanismes ont été mis à profit pour cartographier d'abord le champ de contrainte dans l'ensemble du volume affecté par la microsismicité, puis, dans un second temps, la pression interstitielle dans le même volume.

\section{2}

\section{Intégration des données sismiques et des mesures hydrauliques en forage pour la détermination du champ de contrainte}

Différentes méthodes ont été proposées pour extraire des mécanismes au foyer certaines composantes du champ de contrainte régional (Vasseur et al., 1983; Gephart et Forsyth, 1984; Michael, 1987; Julien et Cornet, 1987; Provost et Houston, 2001).

Toutes ces méthodes supposent:

1) que le champ de contrainte est uniforme dans l'ensemble du volume considéré;

2) que les événements microsismiques affectent des surfaces suffisamment petites pour qu'ils ne perturbent pas le champ de contrainte au voisinage des autres événements;

3) que le vecteur déplacement $d$ est parallèle et de même sens que le cisaillement $\tau$ dans le plan: $\mathrm{d} /|\mathrm{dl} . \tau /| \tau \mid=1$.

De ce fait, étant donné que seule la direction de la composante de cisaillement dans le plan est contrainte, et non son amplitude, l'inversion de ces données ne fournit que quatre composantes du tenseur de contrainte moyen dans le volume considéré:

$$
\underline{\sigma}=\sigma_{1} \underline{I}+\left(\sigma_{3}-\sigma_{1}\right) \underline{I}
$$

où $\underline{\sigma}$ est le tenseur de contrainte moyen dans le volume considéré, $\sigma_{1}, \sigma_{2}$ et $\sigma_{3}$ les valeurs principales de $\sigma$ I le tenseur unité et $I$ un tenseur ayant les mêmes directions principales que $\sigma$ (caractérisées par les trois angles d'Euler) et pour valeurs principales 0 , $\mathrm{R}$ et 1 . avec $R$ un rapport de forme: $R=\left(\sigma_{2}-\sigma_{1}\right) /\left(\sigma_{3}-\sigma_{1}\right)$. L'inversion des mécanismes au foyer permet de déterminer le tenseur $\mathrm{I}$ c'est-à-dire les trois angles d'Euler ainsi que le rapport de forme $\mathrm{R}$.

Yin et Cornet (1994) ont développé une méthode qui permet d'inverser simultanément les résultats des essais HTPF et les directions des vecteurs de glissement pour déterminer le champ de contrainte tel que défini par la relation (2). En d'autres termes, les mécanismes au foyer permettent d'extrapoler à l'ensemble du volume affecté par la sismicité induite les mesures de contrainte obtenues au voisinage immédiat des forages par méthode hydraulique.

\footnotetext{
${ }^{1}$ Rappelons que pour un double couple on définit deux plans nodaux perpendiculaires individualisant deux quadrants pour lesquelles la première arrivée des ondes P est en compression et deux quadrants pour lesquels elle est en extension (Burridge et Knopof, 1964). L'un des deux plans nodaux est le plan de faille mais le seul diagramme de polarité des ondes $\mathrm{P}$ ne permet pas de l'identifier.
}

Les résultats obtenus à partir d'un modèle à 10 paramètres, en négligeant les variations de contrainte dans les directions horizontales, étant donné l'absence de topographie significative et l'homogénéité du massif granitique, sont présentés sur la figure 4. On remarque que la direction verticale est trouvée être principale. Elle correspond à la contrainte principale minimale dans les 200 premiers mètres sous la surface du sol mais devient la direction de la contrainte principale maximale au-dessous de $500 \mathrm{~m}$ de profondeur. De plus les directions des contraintes horizontales tournent dans les premiers $400 \mathrm{~m}$ pour se stabiliser en dessous de cette cote. Ceci illustre ainsi l'impossibilité de déterminer les directions des contraintes tectoniques à partir de mesures trop superficielles.

Les résultats de la figure 4 sont homogènes avec 20 des 22 essais HTPF effectués dans l'intervalle de profondeur dans lequel des événements microsismiques ont été observés (écarts entre valeurs observées et valeurs calculées inférieurs à un écart-type) mais seulement $70 \%$ des mécanismes au foyer.
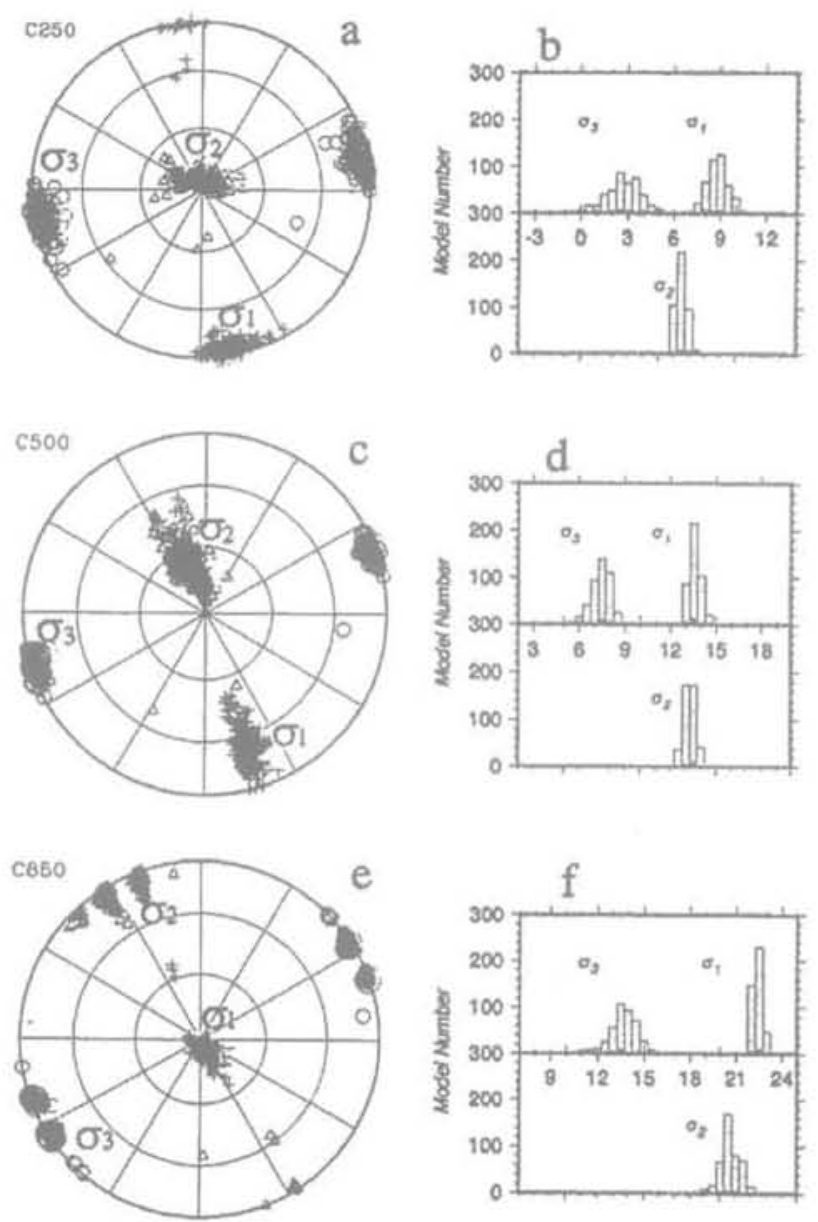

FG. 4 Variations avec la profondeur du champ de contrainte, déterminées en intégrant les données hydrauliques et les mécanismes au foyer (domaine de confiance à $90 \%$ ). 
Nous concluons que les événements microsismiques, étant localisés sur des fractures préexistantes, peuvent, pour certains, échantillonner des zones d'hétérogénéité de contrainte. Il en résulte que l'inversion des seuls mécanismes au foyer peut fournir des résultats biaisés, ainsi que cela a été observé puisqu'un écart systématique de 25 à $30^{\circ}$ a été observé entre les résultats obtenus, lorsque chaque ensemble de données a été inversé séparément.

L'inversion simultanée décrite ci-dessus présente deux intérêts : d'une part, elle identifie les mécanismes hétérogènes, d'autre part, pour les mécanismes homogènes, elle identifie le plan nodal qui correspond au plan de faille. Cette information peut être alors mise à profit pour déterminer la surpression requise pour induire les glissements observés ce qui, de facto, transforme les événements microsismiques homogènes en capteurs de pression distribués dans l'ensemble du nuage microsismique (Cornet et Yin, 1995).

\section{3}

\section{Les événements microsismiques comme piézomètres distribués loin des forages}

L'origine de la sismicité induite est clairement liée à l'augmentation de pression interstitielle. Il est généralement admis que la stabilité des fractures préexistantes est contrôlée par le frottement le long de ces surfaces et que, pour des contraintes normales supérieures à quelques Mégapascals, ce frottement est bien décrit par le critère de Coulomb exprimé en contraintes effectives:

$$
|\tau| \leq \mu\left(\sigma_{n}-\beta p\right)+C_{0}
$$

où $\mu$ et $C_{0}$ sont respectivement le coefficient de frottement et la cohésion, $p$ est la pression interstitielle et $\beta$ un terme caractéristique de la loi de contrainte effective considérée, qu'il est habituel de prendre égal à 1 pour les lois de frottement. Il est fréquemment admis que $\mathrm{C}_{i}$ est négligeable pour les fractures préexistantes mal recimentées. Par ailleurs, la pression interstitielle est égale à $\mathrm{P}_{0}+\mathrm{dp}$, où $\mathrm{P}_{0}$ est la valeur de la pression interstitielle initiale, avant toute injection, et dp est l'augmentation de pression induite par les essais d'injection.

Connaissant le champ de contrainte régional et l'orientation du plan de glissement, il est aisé de calculer $|\tau|$ et $\sigma_{n}$. Par ailleurs, tous les forages réalisés sur le site ont montré que le niveau d'eau est partout très proche de la surface du sol de sorte que la pression interstitielle initiale est donnée par le poids de la colonne d'eau à la profondeur considérée. Les valeurs de la perturbation dp requise pour induire des glissements pour les divers événements microsismiques observés ont alors été calculés pour diverses valeurs de $\mu$ et $\beta$ dans l'équation (4). II a été possible de définir les domaines de valeurs admissibles pour ces deux paramètres étant donné que dp doit rester positif d'une part, et inférieur à la pression en tête de puits d'autre part. Il apparaît ainsi que le terme $\beta$ doit être égal à 1 sans quoi les valeurs requises pour induire des glissements sont supérieures à la pression d'injection, même pour un coefficient de frottement aussi petit que 0,4 . Une fois adoptée la loi de contrainte effective classique, il apparait que le coefficient de frottement doit rester compris entre 0,6 et 1, ce qui est tout à fait cohérent avec ce qui est connu par les essais de laboratoire.
Nous avons alors reporté pour tous les événements de profondeur inférieurs à $750 \mathrm{~m}$ la valeur de dp en fonction de la distance la plus courte à la zone d'injection dans le forage (Fig, 5). En effet, dans ce domaine de profondeur, les événements se distribuent en deux zones principales relativement planes pour lesquelles on peut définir un pendage et un azimut. La première recoupe le forage au voisinage de $500 \mathrm{~m}$ et la seconde au voisinage de $620 \mathrm{~m}$. Pour la zone la moins profonde, la valeur de la pression dp reste très proche de la valeur de la pression d'injection, même à plus de $150 \mathrm{~m}$ du forage, tandis que dans la zone inférieure on observe une chute régulière de pression avec la distance.
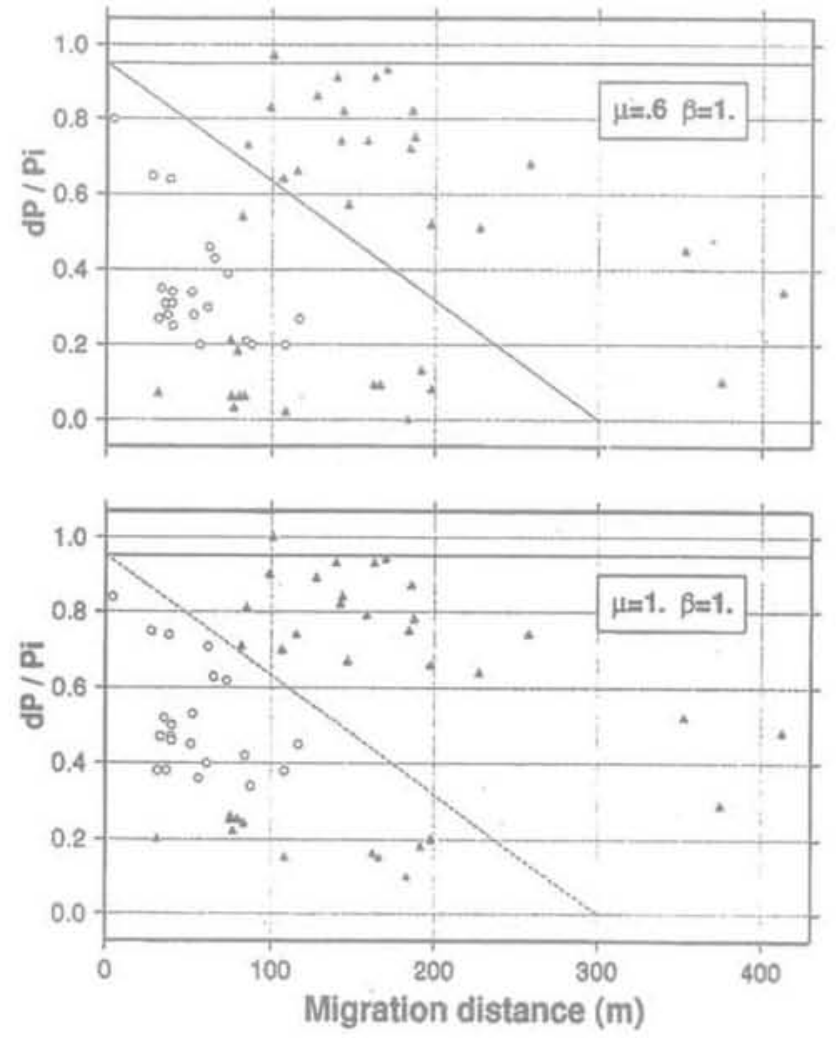

FIG.5 Cartographie des variations de pression induites par les stimulations hydrauliques. Les triangles pleins correspondent aux événements de la zone supérieure identifiée vers $500 \mathrm{~m}$ (Fig. 3) tandis que les ronds correspondent aux événements de la zone fracturée vers $650 \mathrm{~m}$.

Ces résultats sont tout à fait cohérents avec les débitmétries qui montrent que la zone la plus profonde est une zone majeure d'écoulement tandis qu'aucun écoulement notoire n'est détecté dans la zone la plus superficielle. Cette dernière correspond donc à une zone connectée au forage mais non connectée au réseau de fractures significatif du point de vue hydraulique pour le massif.

Cet exemple illustre ainsi qu'à condition de disposer d'informations complémentaires en forage, il est possible de tirer parti des événements microsismiques pour d'une part préciser le champ de contrainte loin des forages, d'autre part obtenir des informations sur la pression interstitielle locale. Elle ne suppose pas que 
l'ensemble du massif soit proche de l'équilibre mais seulement que certaines fractures sont réactivées du fait tant de leur orientation que de leur connectivité au réseau de fractures hydrauliquement significatif.

On retiendra enfin que la sismicité induite n'est pas la preuve d'un débit élevé local mais seulement d'une augmentation de pression. Nous verrons dans l'exemple suivant que cette sismicité induite peut, dans certains cas, renseigner sur les écarts qui existent entre le champ de contrainte naturel et les conditions d'équilibre du massif.

\section{3}

\section{Sismicité induite}

et conditions d'équilibre dans la croûte:

\section{les résultats de Soultz-sous-Forêts}

\section{1}

\section{Contexte observationnel: les essais hydrauliques de 1993}

Du fait de la forte anomalie géothermique locale, la région de Soultz-sous-Forêts, située à environ $40 \mathrm{~km}$ au nord-est de Strasbourg, dans le fossé rhénan, a été choisie pour y implanter un site européen d'expérimentation géothermique en vraie grandeur (Baria et al., 1995; Gérard et al., 1997). Il s'agit d'y développer une méthode permettant d'extraire la chaleur des granites rencontrés sous la couverture sédimentaire à partir de $1500 \mathrm{~m}$ de profondeur.

Les observations décrites ci-après ont été obtenues à l'occasion des premiers essais de reconnaissance des propriétés hydrauliques entrepris en septembre 1993, lors d'injections à débit croissant, entre les cotes 2850 et $3400 \mathrm{~m}$ (Fig. 6) dans le forage GPK1. Tous les jours, durant les essais d'injection, une débitmétrie était réalisée afin d'identifier l'évolution des zones d'écoulement au niveau de leur intersection avec le forage (Cornet et Jones, 1994).

Par ailleurs, des diagraphies d'imagerie acoustique réalisées avant et après les essais ont permis d'examiner les altérations induites à la paroi du forage par cette injection (Cornet et al., 1997). Ces diagraphies ont notamment permis d'identifier une fracture verticale de plus de $40 \mathrm{~m}$ de haut, orientée dans la direction moyenne $\mathrm{N} 175^{\circ} \mathrm{E}$. Cette fracture qui n'était pas présente avant les essais et qui n'était pas le site d'écoulement significatif a été interprétée comme résultant d'une rupture thermique (la température initiale était de $145^{\circ} \mathrm{C}$ et la température en fin d'injection de $33^{\circ} \mathrm{C}$ ). Étant donné qu'à $2000 \mathrm{~m}$ de profondeur une fracture verticale également nord-sud avait été créée lors d'essais de fracturation hydraulique, il a été conclu que le forage est parallèle à une direction principale et que la composante maximale horizontale est approximativement nord-sud sur ce site. Ces résultats ont été confirmés par la suite à l'occasion d'observations effectuées dans le forage GPK2 de $3800 \mathrm{~m}$ de profondeur et situé à $500 \mathrm{~m}$ de GPK1 (Brudy et Zoback, 1999).
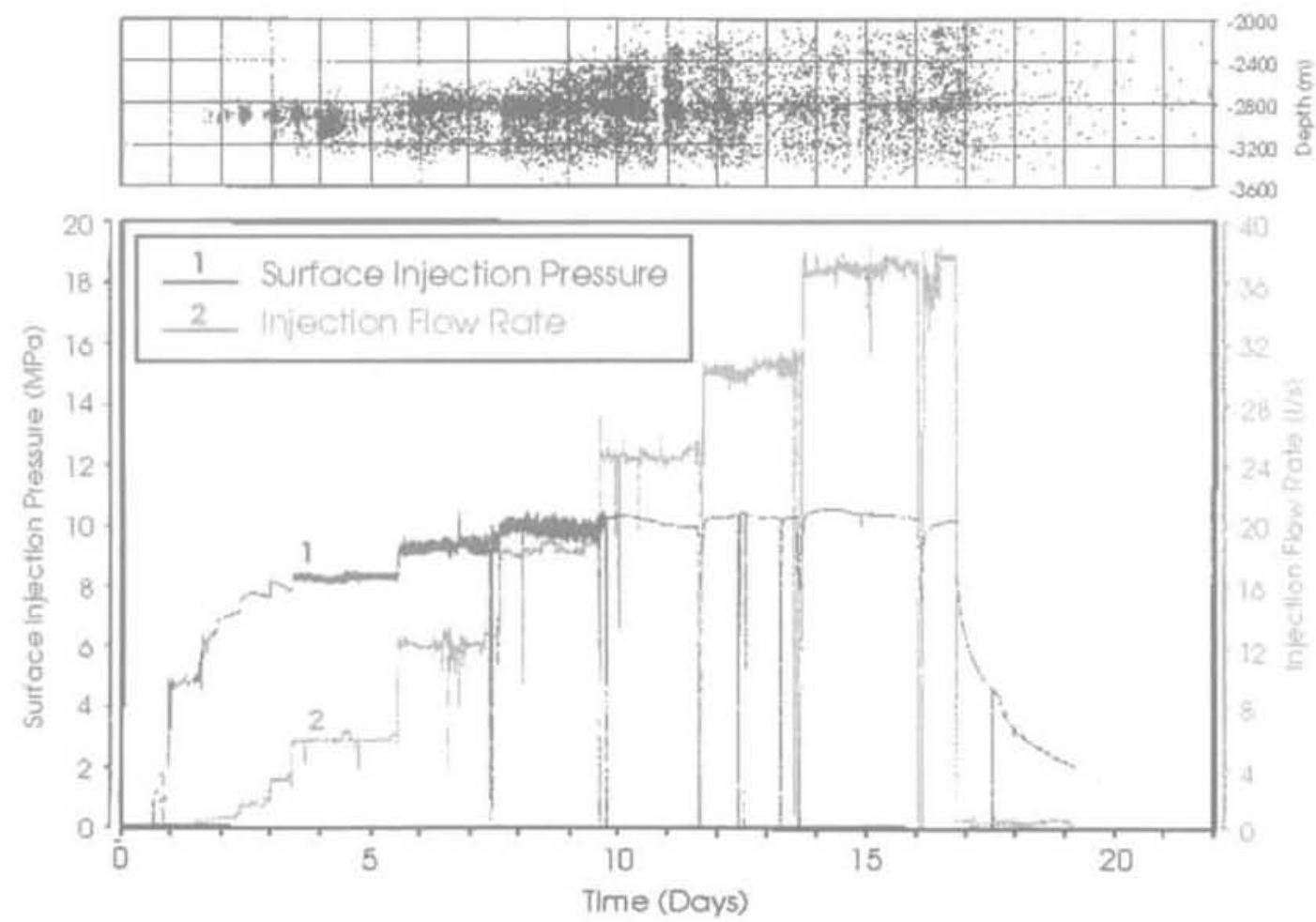

FG.6 Débits et pression d'injection en tête du puits GPK1 durant les premiers essais de reconnaissance à Soultz (septembre 1993). 
Les diagraphies d'imagerie acoustique ont également permis de détecter des mouvements de cisaillements (Fig. 7) dans la partie haute de la zone d'injection, entre 2850 et $2950 \mathrm{~m}$ de profondeur. Pour sept de ces mouvements, les déplacements ont été suffisamment importants pour pouvoir être mesurés. Ils varient de $5 \mathrm{~mm}$ à plus de $4 \mathrm{~cm}$ pour les plus importants (tableau I). Il n'est pas inintéressant de souligner que ces mouvements ne sont pas homogènes avec un champ de contrainte de direction monotone et il est permis de penser que les premiers glissements ont induit des perturbations locales de la contrainte qui ont influencé les suivants. Ce point sera repris lors de la discussion des résultats microsismiques.

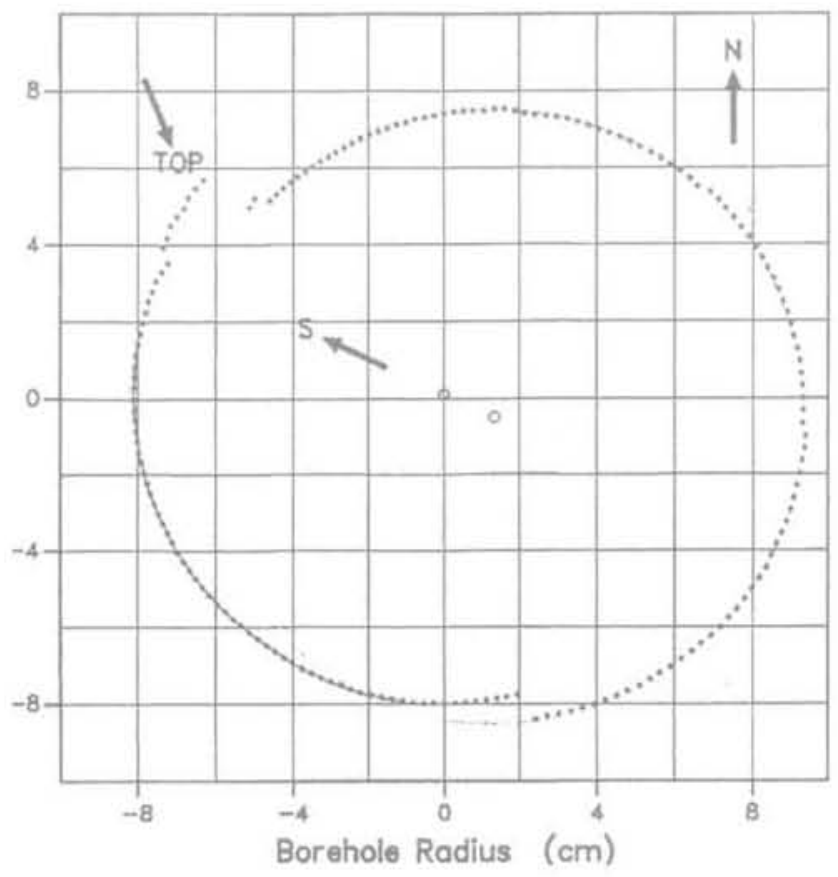

FIG.7 A l'aide d'imagerie acoustique, identification de mouvements de cisaillements dans le forage GPK1 à l'issue des essais d'injection (on exploite ici les mesures de temps de parcours entre loutil et la paroi).

\section{2}

\section{Des apports de la microsismicité induite pour la détermination du champ de contrainte régional}

Les essais ont été accompagnés d'un suivi microsismique. Un ensemble de 3 sondes accélérométriques, comportant trois composantes chacune, ainsi qu'un hydrophone, avait été mis en place dans le granite à la base des sédiments par la Camborne School of Mines, afin d'obtenir une bonne localisation des événements (Jones et al., 1995). Un réseau d'une quinzaine de stations avait été déployé en surface par l'IPG de Strasbourg pour déterminer les mécanismes au foyer des événements les plus forts (Helm, 1996). Si le réseau accélérométrique ancré dans le granite a enregistré de l'ordre de 11000 événements au cours des essais d'injection, le réseau sismique de surface n'a, lui, détecté que quelque 130 événements, de magnitude comprise entre 0,5 et 1,9. Ceci démontre, s'il en était besoin, le rôle de l'atténuation et donc la nécessité de disposer de capteurs profonds si l'on souhaite bien résoudre la forme du nuage microsismique et donc identifier avec précision les zones d'augmentation de pression.

On a reporté sur la figure 6 la profondeur des événements observés en fonction du temps. On remarque tout d'abord que la pression doit atteindre $5 \mathrm{MPa}$ pour qu'apparaissent les premiers événements microsismiques, ceux-ci intervenant dans le haut de la zone d'injection. Puis, si la croissance du nuage a tendance à intervenir vers le bas tant que la pression reste comprise entre 5 et $10 \mathrm{MPa}$, une migration vers le haut intervient lorsque la pression d'injection se stabilise aux alentours de $10 \mathrm{MPa}$, indépendamment du débit d'injection. Cette stabilisation suggère que la pression est devenue égale ou légèrement supérieure à la contrainte principale minimale effective au voisinage de la partie supérieure de la zone d'injection dans le forage. Nous ne débattrons pas plus avant de cette valeur étant donnée la nécessité de prendre en compte les effets thermiques dans cette analyse, ce qui nous entrainerait au-delà de notre propos.

\subsection{4}

De la forme du nuage microsismique vis-à-vis des directions principales de contrainte

Sur la figure 8 on indique la cartographie de l'ensemble des événements observés par les accéléromètres de fond, la coupe verticale étant orientée dans la direction d'allongement majeur du nuage. Sur la figure 9 sont représentées les projections dans un plan horizontal des événements observés dans divers intervalles de profondeur. On remarque clairement un changement d'orientation dans la direction privilégiée du nuage, selon les tranches de profondeur. Seule la tranche centrée sur 2900 m montre une orientation nord-sud, les plus profondes montrant une orientation proche de la direction $\mathrm{N} 150^{\circ} \mathrm{E}$.

Comme nous l'avons déjà vu, la direction nord-sud a été établie être la direction de la contrainte maximale horizontale dans le massif. Or, l'intervalle de profondeur $2800-2900 \mathrm{~m}$ est aussi l'intervalle dans lequel la stimulation a été la plus efficace, ainsi que le montrent

\begin{tabular}{lcccccc}
$\mathrm{Z}(\mathrm{m})$ & $\beta$ & $\alpha$ & $\lambda$ & $\mathrm{A}(\mathrm{cm})$ & $\varepsilon_{2}$ & $\varepsilon_{\boldsymbol{\Lambda}}(\mathrm{cm})$ \\
\hline 2966 & 105 & 84 & 110 & 4,7 & 5 & 0,7 \\
2867 & 259 & 62 & 304 & 2,2 & 3 & 0,1 \\
2976 & 269 & 61 & 218 & 0,8 & 15 & 0,2 \\
2887 & 298 & 75 & 271 & 0,85 & 8 & 0,3 \\
2973 & 273 & 78 & 198 & 0,4 & 10 & 0,06 \\
2925 & 48 & 86 & 99 & 4,3 & 13 & 1,3 \\
\hline
\end{tabular}




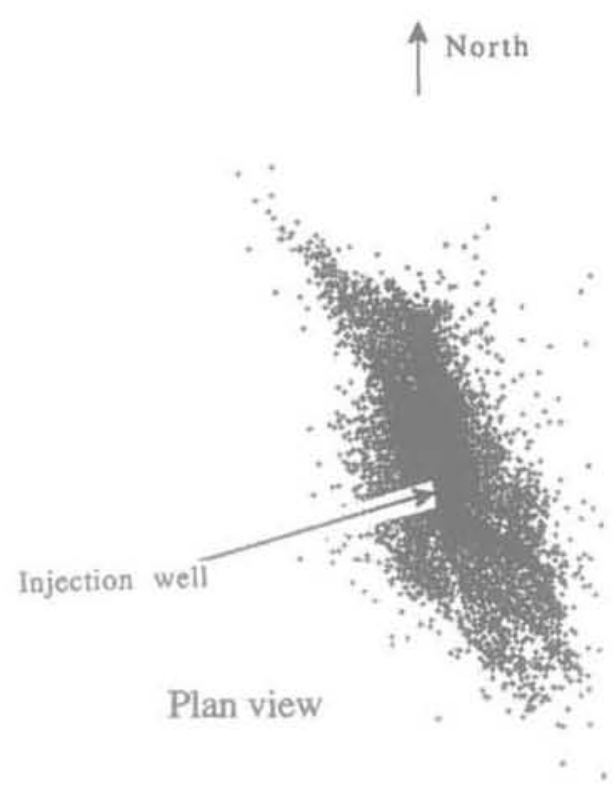

250500

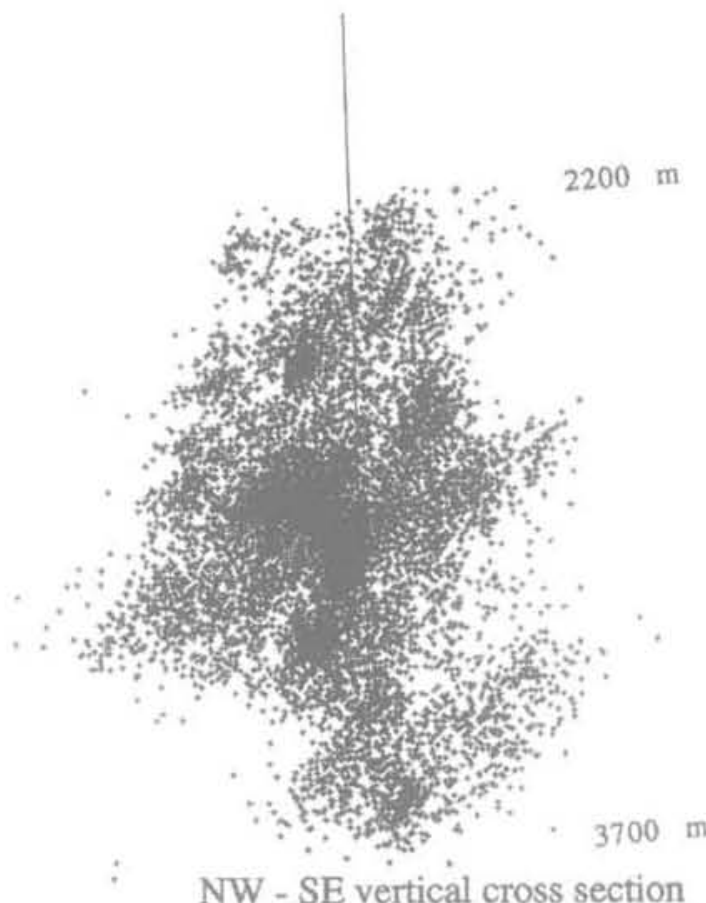

NW - SE vertical cross section horizontal; à droite projection dans un plan vertical orienté dans la dírection d'extension horizontale maximale du nuage.

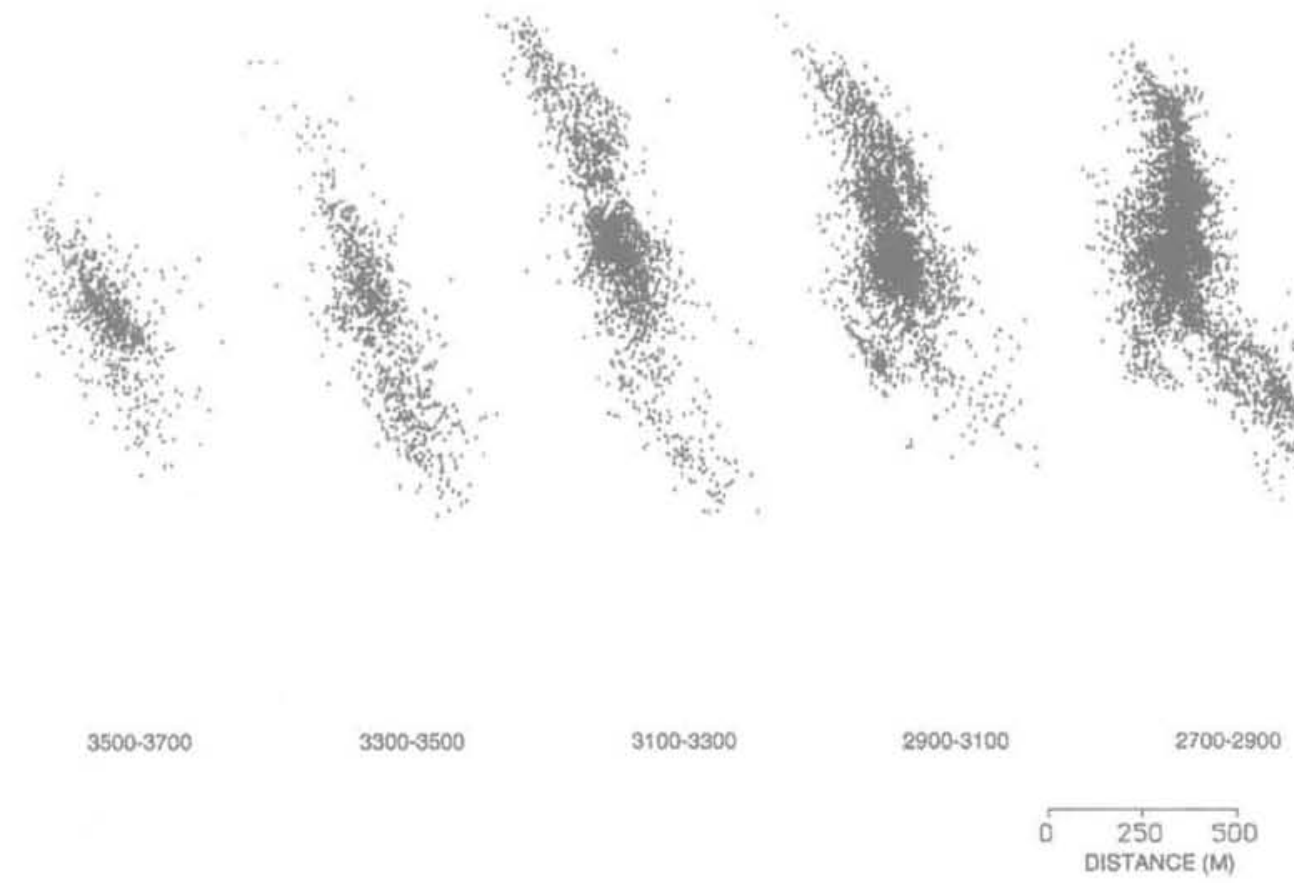

FIG.9 Variations d'orientation du nuage microsismique avec la profondeur. Les événements sont projetés dans le plan horizontal pour diverses tranches de profondeur. 
les débitmétries. On remarque donc que les structures les mieux stimulées s'organisent de façon quasi perpendiculaire à la direction de la contrainte principale minimale et donc que la pression d'injection est contrôlée par l'amplitude de la contrainte principale minimale effective locale.

On conclut également que l'orientation privilégiée du nuage pour les autres tranches de profondeur n'est pas la direction de la contrainte maximale horizontale mais se trouve orientée à peu près à $30^{\circ}$ de cette dernière. Elle correspond à l'orientation de la zone de rupture que l'on attendrait si l'on retenait un critère de Coulomb pour la rupture dans ce massif. On remarque cependant que, loin du forage, la forme du nuage microsismique est plus variée, soulignant probablement la présence de structures préexistantes. Un des objectifs majeurs des travaux en cours est de préciser, à partir des localisations des événements microsismiques, la géométrie de ces structures préexistantes.

Sur la figure 6, on remarque que la pression d'injection doit atteindre $5 \mathrm{MPa}$ pour qu'apparaissent les premiers événements microsismiques. Puis le nuage se développe autour du forage de façon relativement symétrique. Ce n'est que lorsqu'il atteint 7 à $8 \mathrm{MPa}$ que l'enveloppe du nuage microsismique semble se structurer en fonction d'un mode de rupture à grande échelle. A partir de ce seuil de pression, le nuage microsismique est uniformément distribué sur une hauteur de plus de $600 \mathrm{~m}$ et où on y discerne assez nettement les structures préexistantes plus petites, quand elles existent. Il semble donc que les conditions d'équilibre du massif, sur ce site, ne sont pas tant contrôlées par de grandes failles préexistantes orientées de façon optimale que par la résistance du matériau continu équivalent défini au moins à l'échelle des quelques centaines de mètres d'extension verticale du nuage microsismique.

Ainsi, qu'il a déjà été noté, la sismicité induite témoigne d'une augmentation de pression locale. Il a été proposé (Shapiro et al., 1999 et 2000) d'exploiter la vitesse de croissance du nuage microsismique pour déduire de cette vitesse de croissance la perméabilité du massif, à grande échelle. Nous soulignerons ici que si cette croissance est effectivement apparemment contrôlée par la perméabilité du massif pour les pressions inférieures à 7 ou $8 \mathrm{MPa}$, pour les pressions d'injection supérieures la croissance du nuage reflète essentiellement le développement d'un processus de rupture et ne doit pas être mis en relation avec la perméabilité intrinsèque du milieu.

\subsection{8}

\section{De la mise en évidence de mouvements asismiques et de l'exploitation des mécanismes au foyer pour déterminer le champ de contrainte régional}

Ainsi qu'il a été mentionné ci-dessus, ces injections ont induit un certain nombre de mouvements de cisaillement détectés dans le forage, dont certains pluricentimétriques selon les mesures par imagerie acoustique (tableau I).

Si l'on suppose le milieu élastique et les surfaces de glissement planes et de pourtour circulaire, la relation entre la chute de contrainte moyenne $\Delta \sigma$, le déplacement D (dislocation supposée uniforme sur toute la surface) et le rayon a de l'aire de rupture est bien connue, si l'on suppose un comportement élastique pour la roche (Kanamori et Anderson, 1975):

$$
\Delta \sigma=7 \pi / 16 * G^{*} \mathrm{D} / \mathrm{a}
$$

où $\mathrm{G}$ est le module de cisaillement du matériau. Le moment sismique $\mathrm{M}_{0}$ du double couple est fonction de l'aire $S$ de la surface de rupture et de l'amplitude de la dislocation $\mathrm{D}$ :

$$
M_{0}=G^{*} S^{*} D
$$

Par ailleurs, certains auteurs (Pearson, 1981; Majer et McEvilly, 1979) ont développé des relations empiriques entre le moment sismique et la magnitude des séismes induits par des injections d'eau. Les données utilisées proviennent d'essais d'injection dans le granite de Fenton Hill, à Los Alamos, et du champ des Geysers, en Californie. Elles sont très comparables aux conditions des essais de Soultz et ont été retenues pour ce dernier site:

$$
\log \left(M_{0}\right)=17,27+0,77 \mathrm{M}
$$

où $\mathrm{M}$ est la magnitude estimée à partir de la durée du signal.

Il apparait ainsi qu'en assimilant les événements microsismiques observés à des dislocations, il est possible d'obtenir une relation entre les dimensions de la source, la chute de contrainte, l'amplitude de la dislocation et la magnitude observée.

Le module de cisaillement a été évalué à partir des vitesses sismiques déterminées pour ce milieu. Par ailleurs, connaissant la profondeur de l'événement on en déduit la valeur de la contrainte verticale à cette cote, valeur qui correspond à la contrainte principale maximale En effet, les mécanismes au foyer correspondent en majorité à des mécanismes en faille normale (contrainte principale maximale verticale) avec, cependant, de très nombreux décrochements (contrainte principale maximale horizontale). Ces mécanismes suggèrent donc que la composante principale verticale et la composante principale maximale horizontale sont très proches l'une de l'autre.

Une sous-estimation de la contrainte principale minimale peut être obtenue en considérant que cette dernière doit satisfaire les conditions d'équilibre du massif si l'on suppose qu'il existe toujours des fractures préexistantes orientées dans la direction critique. On obtient ainsi une surestimation de la chute de contrainte maximale associée aux événements considérés.

Ces considérations amènent à conclure que si les glissements pluri-centimétriques observés à la paroi du forage avaient été associés à des ruptures sismiques uniques, la magnitude correspondante des événements aurait été comprise entre 3,5 et 4,2 , selon la valeur de la chute de contrainte moyenne retenue. Or les observations sismiques révèlent que la magnitude maximale observée n'a pas dépassé 1,9.

La question se pose de savoir si les glissements observés pourraient résulter d'un cumul de N mouvements sismiques plus petits. Une estimation des déplacements, associés aux événements sismiques les plus forts, montre qu'il faudrait que tous les événements interviennent sur la même surface pour que soit atteint l'ordre de grandeur des déplacements observés. Or ces événements sont dispersés dans l'ensemble de l'espace d'une part, d'autre part il existe plusieurs zones pour lesquelles des mouvements pluri-centimétriques ont été observés.

Il est donc conclu que les injections d'eau ont induit des mouvements asismiques. Ceux-ci ont per- 
turbé localement le champ de contrainte ce qui expliquerait bien pourquoi l'inversion des mécanismes au foyer n'a pas donné comme orientation de la direction de contrainte principale maximale horizontale la direction nord-sud déjà décrite, mais une orientation $\mathrm{N} 125^{\circ} \mathrm{E}$.

Ces résultats confirment les résultats du Mayet-deMontagne à savoir que l'inversion des seuls mécanismes au foyer peut déboucher sur des résultats biaisés du fait d'hétérogénéités locales de contrainte au voisinage des foyers de certains microséismes. Il convient donc toujours d'associer ce type de données à d'autres observations indépendantes, pour une détermination du champ de contrainte régional, afin d'éliminer les événements non représentatifs. Resterait à définir rigoureusement ce que l'on dénomme " champ de contrainte régional $n$, mais ce point ne sera pas abordé ici.

\subsubsection{3}

\section{De la détection des directions d'anisotropie de vitesse sismique et de leur relation} avec les directions de contraintes principales régionales

Lorsqu'une onde S se propage dans un milieu anisotrope, sa vitesse de propagation dépend de la direction de polarisation de l'onde vis-à-vis des axes de symétrie du matériau. De ce fait les milieux anisotropes décomposent généralement les ondes $\mathrm{S}$, donnant ainsi lieu au phénomène de biréfringence, c'est-à-dire à deux arrivées d'ondes S pour une même source.

On distingue souvent trois types d'anisotropie:

1) I'anisotropie liée à l'orientation préférentielle des minéraux;

2) l'anisotropie de stratification liée à des alternances de couches dont l'épaisseur doit rester petite devant les longueurs d'onde considérée pour satisfaire les conditions d'homogénéité :

3) l'anisotropie de fissuration liée à une orientation préférentielle des microfissures.

De nombreuses questions restent posées sur la dimension critique des discontinuités qui influencent les vitesses de propagation (Crampin et Lowell, 1991). Mais, il est souvent admis que le champ de contrainte, en influençant la géométrie des microfissures, induit une anisotropie dans le milieu. Pour une distribution aléatoire des microfissures, celles qui sont perpendiculaires à la direction de la contrainte principale maximale sont plus is fermées $x$ que celles qui sont perpendiculaires à la direction de la contrainte principale minimale. Ainsi, les ondes S polarisées perpendiculairement à la direction de contrainte maximale doiventelles être plus rapides que les ondes polarisées perpendiculairement à la direction de la contrainte principale minimale. Ces résultats ont été bien vérifiés au laboratoire par Nur et Simmons (1969) et de nombreux travaux ont été réalisés au cours des vingt dernières années pour détecter la biréfringence des ondes S sur le terrain (voir par exemple Bouin et al., 1996; Gamar et al., 2000, pour les résultats obtenus dans le rift de Corinthe). Pour cela, il est, bien sûr, nécessaire d'enregistrer les trois composantes du signal sismique à un pas d'échantillonnage élevé afin de détecter des changements de directions de polarisation éventuels et les instants précis de ces changements de direction.

Cependant plusieurs facteurs contrôlent la polarisa- tion des ondes $\mathrm{S}$, notamment au voisinage des interfaces entre des matériaux de nature différente, et donc particulièrement au voisinage de la surface du sol, du fait des ondes réfléchies. Il est ainsi nécessaire de s'assurer que l'angle d'incidence de l'onde vis-à-vis de l'interface considéré est inférieur à une certaine valeur qui dépend des vitesses de propagation dans chacun des milieux (Crampin et Lovell, 1991). Afin de s'assurer que la polarisation observée n'est pas liée à des effets locaux, elle est étudiée pour un large domaine de longueurs d'onde, en prenant soin de satisfaire les conditions satisfaisantes d'observation (ne pas être en champ proche, c'est-à-dire considéré des distances sourcerécepteur impliquant plusieurs longueurs d'onde et ne considérer que des longueurs d'onde nettement plus grandes que la source, ne pas prendre en compte les ondes de surface et donc s'assurer que les phases détectées correspondent bien à des ondes de volume, etc.).

A Soultz, théoriquement les trois sondes accélérométriques auraient dû permettre d'étudier la biréfringence. En fait une des sondes était implantée dans les sédiments, juste au-dessus du granite, à proximité d'une faille majeure, ce qui perturbe considérablement la polarisation du signal. De ce fait seules deux des sondes étaient disponibles pour ce type d'analyse. L'attention s'est portée sur le volume dans lequel les mouvements asismiques ont été observés, soit une sphère de $100 \mathrm{~m}$ de rayon centrée sur le haut de la zone d'injection. Dans ce volume, un ensemble de 14 microséismes a été également vu sur le réseau de surface. Cet ensemble a été considéré pour l'analyse, ainsi que huit événements d'un multiplet, c'est-à-dire d'événements très similaires par leur contenu fréquentiel et probablement induits par la même source (Gaucher, 1998; Gaucher et al., 1998). De tels multiplets sont intéressants en ce qu'ils permettent d'observer des évolutions éventuelles des propriétés du milieu séparant la source du récepteur, notamment du fait des variations de la pression interstitielle.

Les signaux ont d'abord été intégrés en temps de façon à considérer la vitesse du mouvement et non son accélération, puis ils ont été filtrés avec des filtres passe-bande non déphasant. Les microséismes vus aussi avec le réseau de surface sont très énergétiques et ont été filtrés dans le domaine $50-950 \mathrm{~Hz}$. Les événements du multiplet, beaucoup plus petits que les précédents, ont été examinés dans le domaine $50-250 \mathrm{~Hz}$. Les directions de polarisation telles qu'observées au récepteur ont été ramenées à la source, pour indiquer la diversité des orientations et des distances des signaux traités. On remarque que pour la station la plus profonde (Fig. 10), la direction rapide est à peu près orientée dans la direction nord-sud (N 352 $\pm 10^{\circ}$ E). Pour l'autre station, implantée au voisinage du toit du granite, la direction moyenne est plutôt nord nord-estsud sud-ouest ( $\left.20^{\circ} \pm 10^{\circ} \mathrm{E}\right)$. Il est à souligner que cette station est située au voisinage de l'apex d'un horst local dont l'orientation est celle du fossé rhénan, soit à peu près $\mathrm{N} 20^{\circ} \mathrm{E}$. II semble raisonnable de considérer que cet écart à la direction nord-sud observé plus profondément dans le granite est lié à cette structure géologique locale.

On conclura ici, qu'apparemment, pour ce granite, d'une part une biréfringence des ondes S est observée et que la direction rapide est apparemment cohérente avec la direction de la contrainte principale maximale dans le milieu. 
Premier mouvement S, station 4550

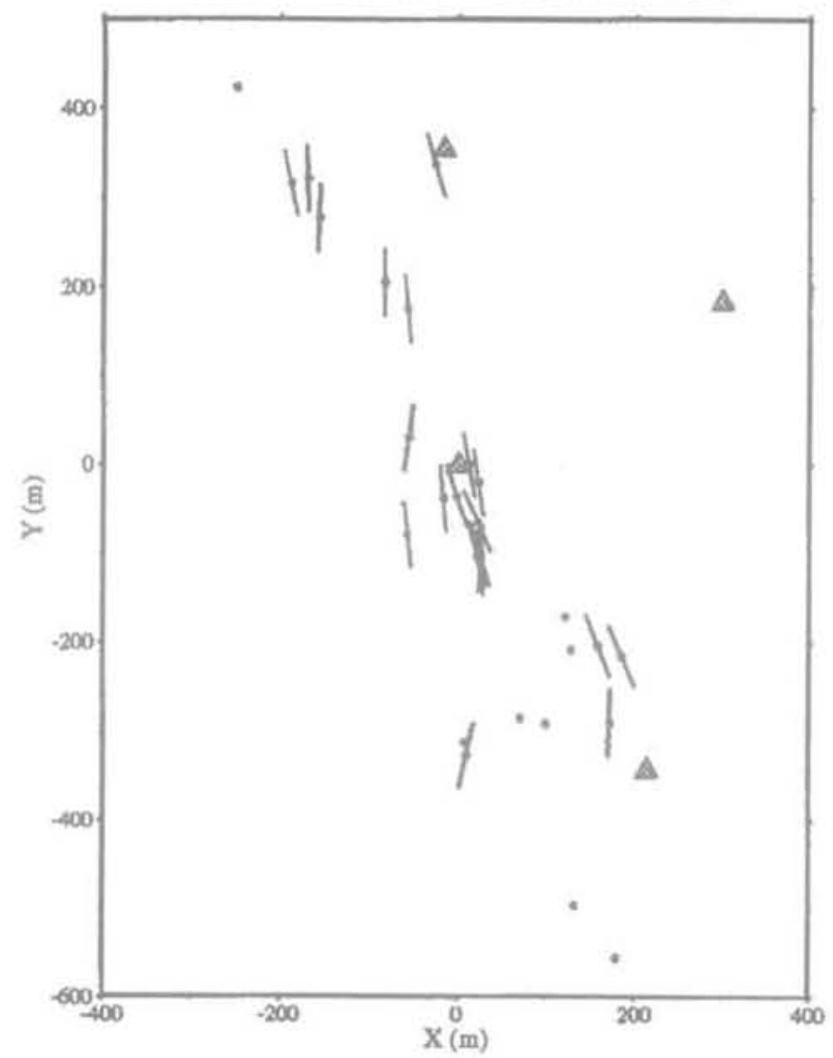

FG. 10 Direction de polarisation $S$ la plus rapide vue à la station la plus profonde dans le granite. Les directions sont représentées à l'emplacement de la source du signal pour indiquer la diversité d'orientation des rais sismiques incidents considérés.

\section{4}

\section{Le champ de contrainte au voisinage d'un secteur asismique de la faille philippine}

Les chapitres précédents ont permis de préciser et illustrer les méthodes permettant de déterminer le champ de contrainte sur de grands volumes. Ils montrent bien la difficulté liée à la détermination de l'ensemble de ses composantes et de leurs variations spatiales. Le cas de l'étude entreprise au voisinage de la faille philippine qui sera discuté maintenant associe des observations sur le champ de contrainte dérivées de données de microsismicité induite, selon les méthodes déjà décrites, et des mesures de déplacement par méthode GPS, ce qui permet d'aborder la question de l'étude in situ de la rhéologie de cette faille.

\section{Contexte sismo-tectonique}

La faille philippine est un grand décrochement sénestre actif, de $1200 \mathrm{~km}$ de long, site de plusieurs séismes de magnitude supérieurs à 7 , notamment dans l'île de Luzon où se trouve Manille (Fig. 11). Son origine, récente (moins de 5 millions d'années), provient de l'obliquité de la convergence entre la plaque philippine et le bloc de la Sonde au niveau de la fosse des Philippines (Fitch, 1972). La faille coupe l'arc volcanique situé en arrière de la subduction, dans la partie centrale de l'archipel (île de Leyte).

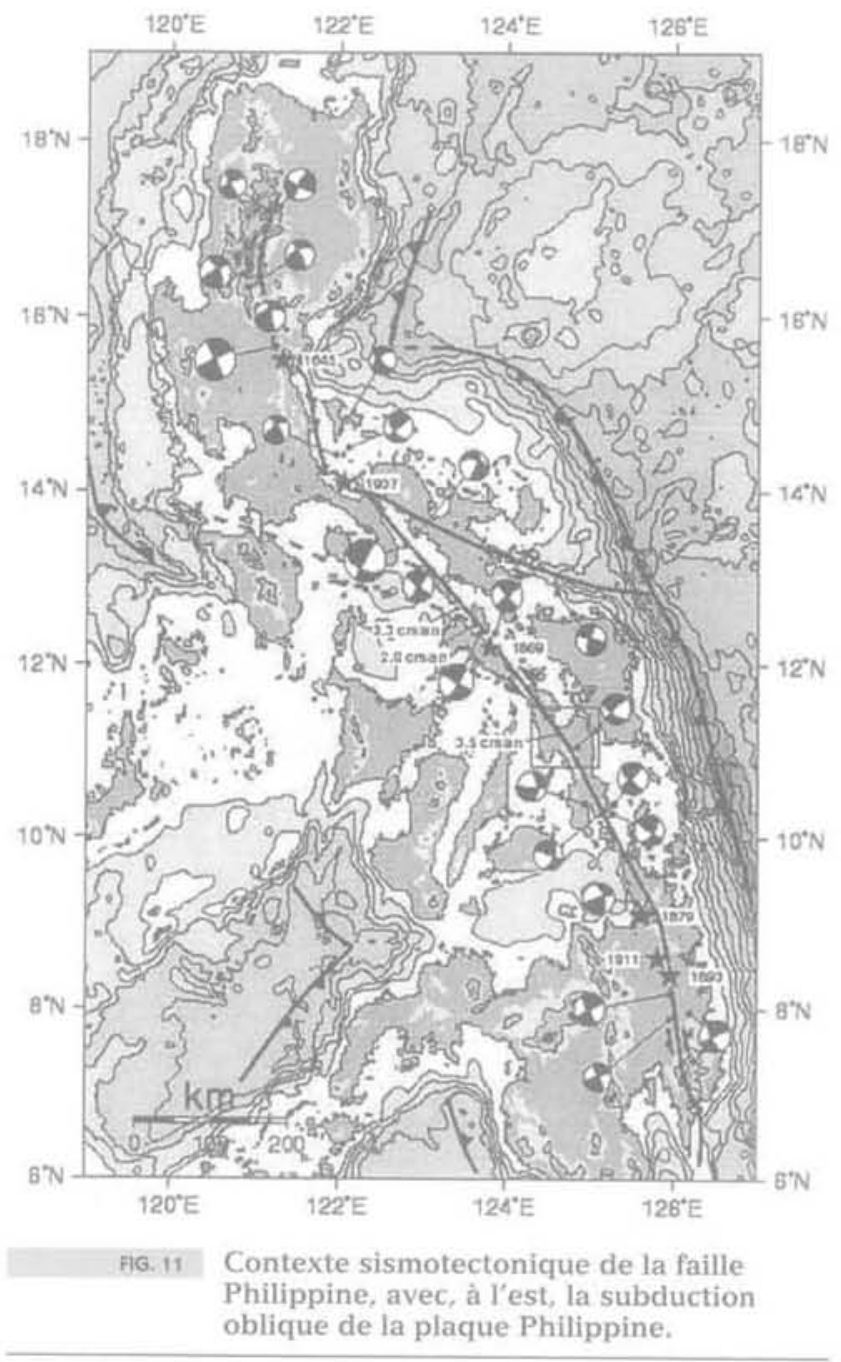

Des observations géologiques et des analyses cinématiques ont montré que la faille philippine est très active dans sa partie centrale, avec des décrochements de 2 à 2,5 cm/an (Barier et al., 1991). Des mesures par la méthode GPS, répétées pendant près de cinq ans (Duquesmoy, 1997), ont montré que la vitesse actuelle atteint $3,5 \mathrm{~cm} / \mathrm{an}$ sur un segment d'au moins $50 \mathrm{~km}$ de long dans le Nord de l'île de Leyte.

Sur le site de Tongonnan, dans le Centre-Nord de l'île de Leyte, la faille recoupe une structure volcanique, site d'un important réservoir géothermique (températures de $300^{\circ} \mathrm{C}$ rencontrées à moins de $2000 \mathrm{~m}$ de profondeur par endroit; la production électrique actuelle dépasse $500 \mathrm{Mw}$ et devrait atteindre $800 \mathrm{Mw}$ quand le champ sera entièrement développé). 
Sur ce site, la faille présente trois segments parallèles (dénommés ouest, central et est) accommodant globalement un décrochement de $2,5 \mathrm{~cm} / \mathrm{an}$. Il n'est pas établi cependant si le décalage entre les vitesses observées à Tongonnan et celles mesurées plus au nord est réel ou simplement provient du fait que les mesures, au niveau de Tongonnan, n'ont pas inclus des points de référence suffisamment éloignés de la faille. Ce point soulève la difficile question d'identifier précisément sur le terrain les limites d'une zone de faille.

Sur l'île de Leyte aucun séisme notoire n'a été relevé depuis que sont disponibles des données quantitatives (1964), si ce n'est pour un séisme de magnitude 5,3 observé dans la partie centre-sud de l'île, en 1993.

\section{2}

\section{Contexte observationnel: les essais hydrauliques de juin 1997}

Le développement du site géothermique a nécessité de réaliser des forages pour la réinjection des saumures produites. Le réservoir étant essentiellement localisé à l'est de la branche centrale de la faille, les ingénieurs géothermiciens avaient espéré pouvoir réinjecter les saumures dans la zone comprise entre les branches ouest et centrale de la faille, suffisamment loin des forages producteurs pour éviter un refroidissement prématuré. Malheureusement, ces forages se sont révélés recoupés des formations beaucoup trop imperméables pour absorber les volumes requis, y compris les forages qui recoupaient les branches ouest et centrale de la faille vers $2000 \mathrm{~m}$ de profondeur. Des essais de fracturation hydraulique classique n'ont pas permis d'améliorer de façon significative les caractéristiques d'injectivité des forages concernés.

Il a alors été décidé de tester la méthode de stimulation des fractures préexistantes décrite ci-dessus, en réalisant une injection à débit croissant par pallier (Cornet et al., 1998), Cette expérience s'est déroulée dans le forage MG2RD qui recoupe la branche centrale de la faille, $1980 \mathrm{~m}$ sous la surface du sol. Le fluide injecté était simplement l'eau de la rivière du site (température de $25^{\circ} \mathrm{C}$ ). L'injection a démarré avec un débit de $10,6 \mathrm{l} / \mathrm{s}$ puis a été ensuite augmentée de $10,6 \mathrm{l} / \mathrm{s}$ tous les deux jours jusqu'à atteindre $53 \mathrm{l} / \mathrm{s}$ et une pression de $9,6 \mathrm{MPa}$ en tête de forage, limite maximale tolérée par les équipements de tête de puits (Fig. 12). Un volume total de $36000 \mathrm{~m}^{3}$ a ainsi été injecté en 12 jours.

Les diagraphies thermiques et débitmétriques ne pouvaient atteindre les zones recoupées par le forage en dessous de son intersection avec la faille, étant donné la diminution de diamètre locale induite par les mouvements de la faille depuis la réalisation du forage en 1993. Ces diagraphies montrent cependant que la quasi-totalité de l'eau a été injectée en dessous de la zone de mesure, c'est-à-dire soit dans la faille, soit plus profondément que son intersection avec le forage.

\section{3}

\section{Activité microsismique et champ de contrainte}

Afin d'obtenir des informations sur les effets de la stimulation hydraulique, un suivi microsismique a été

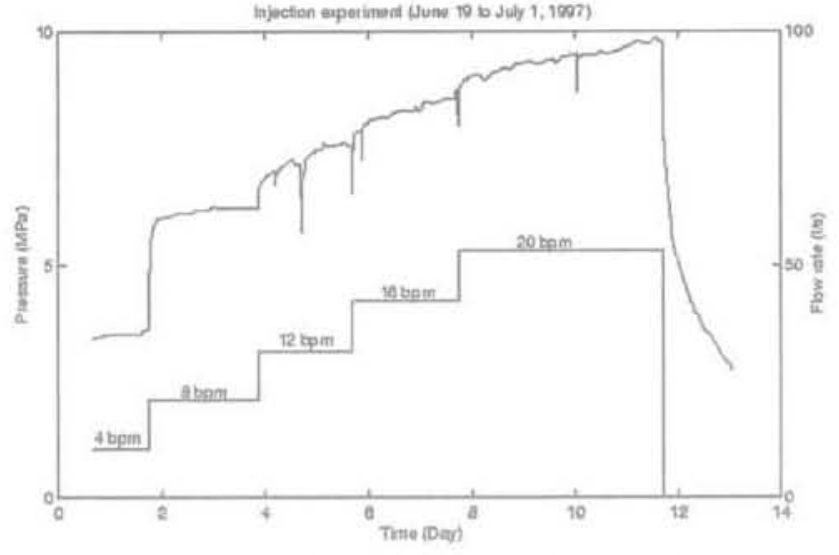

FG. 12

Débits et pression d'injection en tête de puits. Les valeurs de débit ont été calées une fois par jour sur des débitmétries en forage.

assuré. Il s'agissait d'obtenir d'abord un état des lieux avant l'injection, puis d'assurer le suivi de la microsismicité induite pendant et après ces essais.

Le réseau d'observation microsismique a varié dans le temps. De février 1996 à fin juillet 1997, un réseau télémétré, comprenant 7 stations à composante verticale seule, a été déployé. Par ailleurs, un réseau complémentaire télémétré comprenant 7 stations à une composante ainsi qu'un ensemble de 4 stations à 3 composantes ont été déployés en octobre-novembre 1996 et en juin-juillet 1997. La géométrie du réseau avait été adaptée pour suivre la microsismicité associée d'une part aux segments de faille, d'autre part aux opérations d'injection. Toutes les stations étaient simplement posées à la surface du sol. Les réseaux étaient asservis entre eux de sorte que tout événement enregistré sur l'un déclenchait l'enregistrement sur l'autre, assurant ainsi un enregistrement sur au moins 14 stations. Les quatre stations 3-composantes étaient opérées indépendamment, mais avec la même base de temps.

Les données obtenues montrent clairement que les injections réalisées en juin 1997 ont effectivement induit une activité sismique notable (Fig. 13).

Elles montrent également que l'activité microsismique dans le champ géothermique est continuelle du fait de l'exploitation. Très peu d'événements ont été localisés sur la faille au cours des 18 mois de surveillance, une très grande majorité se trouvant à l'est de la faille centrale et à moins de $5 \mathrm{~km}$ de profondeur.

La totalité des événements associés à l'injection dans MG2RD se trouve à l'intérieur du réservoir, en dessous du point d'injection. Aucun événement n'a été observé sur la faille, près de son intersection avec le forage. On est ainsi tenté de conclure que la faille est en fait complètement étanche: si elle avait été le site d'une certaine percolation, la pression interstitielle au voisinage de la faille aurait été perturbée et des événements seraient intervenus, à la manière de ce qui a été observé près du forage dans le réservoir. Ceci montre par ailleurs que les mouvements observés par GPS sur la faille sont essentiellement asismiques. A signaler que le suivi par GPS pendant les essais n'a permis de détecter aucun déplacement. Mais il est vrai que la résolution (5 et $7 \mathrm{~mm}$ pour les coordonnées horizontales, $15 \mathrm{~mm}$ pour les coordonnées verticales) n'était pas très élevée. Un effort a été entrepris pour essayer de déter- 


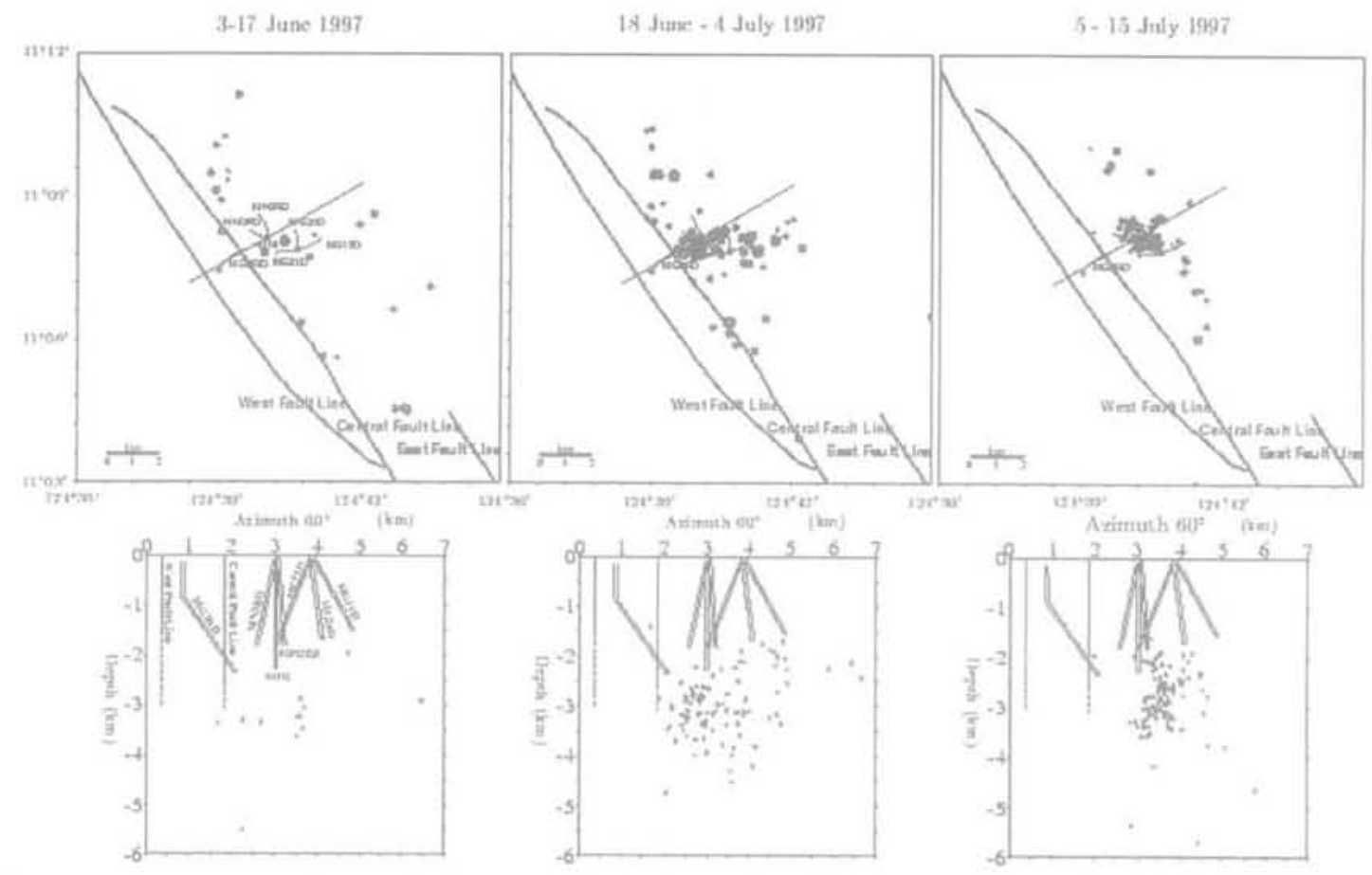

FG. 13 Localisation des événements observés, avant, pendant et après les essais d'injection. Le forage d'injection MG2RD est le forage incliné qui recoupe la faille centraie. Les évérements observés après les essais sont induits par des injections dans des forages voisins.

miner le champ de contrainte au voisinage de la faille à partir de la sismicité induite. Deux types de données sont disponibles: les polarisations des ondes S vues sur les quatre stations 3-composantes et les mécanismes au foyer (interprétés en double couple).

\section{Rósilitats \\ Résultats de la biréfringence}

Les études de biréfringence ont été menées pour les quatre stations 3-composantes (Prioul, 2000). La présence d'ume couche à faible vitesse en surface assure que la plupart des rais sismiques arrivent quasi verticalement aux points d'observation, ce qui autorise un large domaine de localisation possible pour les événements qui peuvent ètre considèrés.

Tous les événements qui présentaient une arrivée de phase S bien impulsive ont été pris en compte, à l'exclusion des signaux dits « émergents »), c'est-à-dire pour lesquels la première arrivée de l'onde S ne sort du bruit sismique que très progressivement. Les stations ayant èté désinstallées entre les deux périodes d'acquisition (octobre-novembre 1996 et juin-juillet 1997), les données ont été regroupées par période d'observation, l'orientation des capteurs n'étant pas les mêmes pour chacune des périodes. Étant donné le caractère impulsif des signaux considérés, il n'a pas été nécessaire de les filtrer.

Les résuitats sont présentés dans le tableau II et sur la figure 14. On y remarque une grande stabilité des résultats. Les trois stations situées à l'est de la faille centrale (G1, G2, G4) donnent des résultats très comparables tandis que la station 3, implantée à l'intersection entre les branches centrales et ouest donne une direction rapide dans une direction quasiment perpendiculaire aux trois autres. Les matériaux impliqués sont pratiquement les mêmes pour toutes les stations mais la station 3 est installée dans une zone d'extension orientée parallèlement aux failles, d'après les observations microtectoniques locales (pull apart observé dans cette zone de relais).

11 apparait ainsi que les directions rapides observées sont liées à la structuration des fractures et microfissures, en liaison avec la présence des failles et non à la structuration minéralogique du matériau. Reste à déterminer si ces directions privilégiées sont induites par le champ de contrainte régional ou par une structuration de la fracturation associée au développement de la faille philippine.

\section{Analyse des mécanismes au foyer}

Durant les deux périodes d'observation, octobre. novembre 1996 et juin-juillet 1997, 18 stations étaient céployées, permettant ainsi de bien contraindre les mécanismes au foyer des événements microsismiques Il s'est avéré que, durant la période octobre-novembre 1996, des essais de production avec réinjection des saumures produites avaient eu lieu à notre insu. La ques.

TABLEAUII Biréfringence des ondes S; orientation (origine au nord, rotations positives vers l'est) des directions de polarisation pour les vitesses les plus rapides. Les stations G1, G2 et G4 sont à l'est de la faille centrale tandis que G3 est à l'intersection des branches centrale et ouest.

\begin{tabular}{|ccccc|}
\hline & G1 & G2 & G3 & G4 \\
\hline Oct.-nov. 1996 & $156^{\circ} \pm 7.7$ & $143^{\circ} \pm 12.5$ & $74^{\circ} \pm 9,3$ & $149^{\circ} \pm 9,7$ \\
Juin-juil. 1997 & $145^{\circ} \pm 9.5$ & $151^{\circ} \pm 9.5$ & $57^{\circ} \pm 11.8$ & $139^{\circ} \pm 8,7$
\end{tabular}




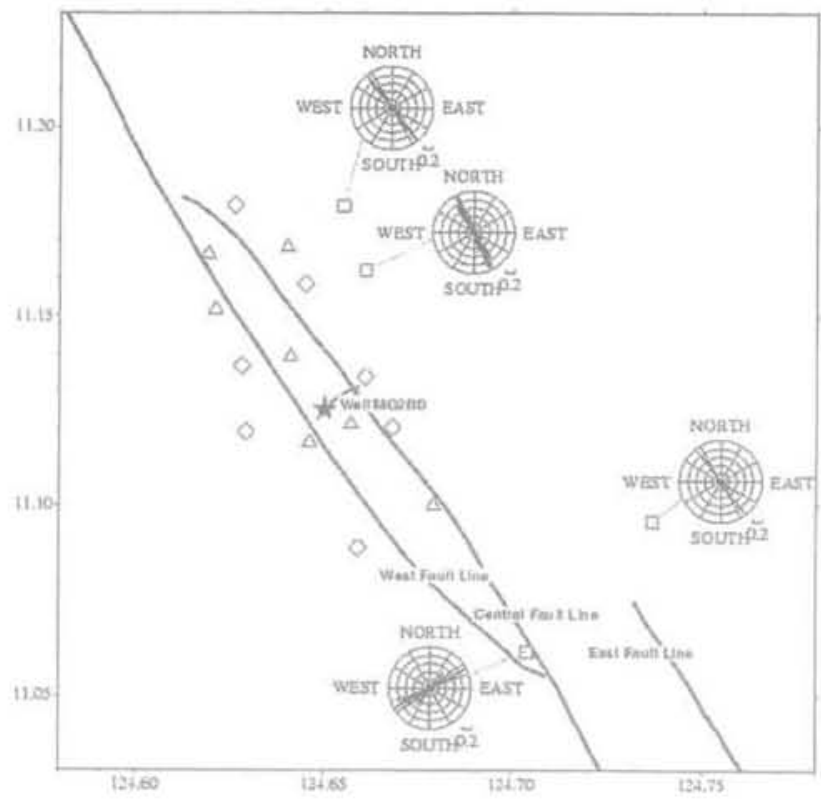

FiG. 14 Biréfringence des ondes S: orientations de la direction de polarisation rapide observées sur les quatre stations à 3 composantes pour la période juin-juillet 1997. Les stations G1 et G2 sont au nordest, la station G4 au sud-est et la station G3 à l'intersection des failles centrale et ouest.

tion s'était posée de l'origine d'un essaim microsismique bien localisé durant cette première période et que nous avons donc pu associer a posteriori à des essais de production.

Ainsi pour les deux périodes un nombre suffisant de mécanismes au foyer a pu être déterminé dans un volume relativement restreint, apte à satisfaire l'hypothèse d'homogénéité du champ de contrainte (23 pour la première période, 32 pour la deuxième pérjode). Il s'avère, de plus, que l'essaim de la première période se trouve au voisinage des stations 3-composantes G1 et G2. ce qui permet de comparer directement les résultats des mécanismes au foyer à ceux de la biréfringence. L'essaim de la deuxième période est, lui, localisé au voisinage du forage MG2RD. Ces données montrent une grande diversité d'orientation des plans nodaux, indiquant ainsi que le champ de fracturation naturelle n'est pas simplement structuré autour d'une seule direction. Ceci suggère que les directions d'anisotropie sont plutôt contrôlées par l'effet des contraintes sur le champ de miocrofissures que par une orientation privilégiée des failles et fractures.

L'inversion a èté effectuée en considérant un modèle à 4 paramètres (tenseur I de l'équation (3)) Dans l'équation (2) supposée représenter les variations de contrainte dans un petit volume, on remarque que, lorsque les variations latérales de contrainte sont négligeables $\left(\alpha_{1}=\underline{\alpha}=\underline{0}\right)$ et que le terme constant devient négligeable devant le gradient vertical (c'est-à-dire pour des profondeurs supérieures à $2000 \mathrm{~m}$ si le terme constant ne fait que refléter les effets associés à la proximité de la surface du sol et que le matériau est hornogène dans l'ensemble du volume considéré), alors le facteur de forme R déterminé caractérise le gradient de contrainte vertical. Dans de telles conditions, la méthode d'inversion reste valable pour des volumes d'extension verticale quelconque.
Les résultats sont indiqués sur les figures 15 et 16. Ces solutions sont mal contraintes, différentes entre elles et de plus incohérentes avec les données de biréfringence. Or les données de biréfringence sont bien ordonnées et ceci pour des sites distants de plus de $12 \mathrm{~km}$ l'un de l'autre. Il semblerait donc que, lả encore, l'inversion des mécanismes au foyer ne permette pas d'identifier correctement les directions de contrainte principales.

Il nous est alors apparu que, comme pour le site de Soultz, il était très probable que des glissements asismiques étaient intervenus, perturbant ainsi localement

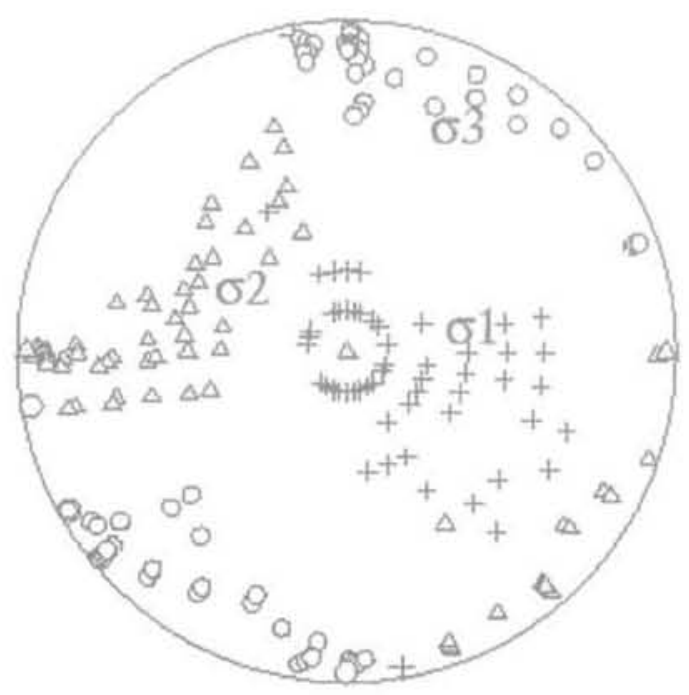

FG. 15 Directions principales de la contrainte régionale déterminées à partir des mécanismes au foyer observés en septembre-octobre 1996. La solution concerne le domaine à $90 \%$ de confiance.

le champ de contrainte et đonc invalidant l'hypothèse 2 requise par l'inversion des mécanismes au foyer. Si un glissement intervient au voisinage d'un événement, le champ de contrainte observé n'est pas le champ de contrainte original et l'évènement correspondant ne peut être inclus dans l'inversion. Ainsi, en négligeant les perturbations de contrainte associées à chaque èvénement on peut être amené à biaiser la détermination du tenseur de contrainte original.

Supposant que ces mouvements asismiques avaient été accompagnés de petits événements locaux, la collection de données disponibles a été redéfinie en ne retenant que des événements indépendants entre eux. Nous avons repris l'ensemble des données disponibles depuis le début de la mise en place du premier réseau d'observation (février 1996) et avons associé à chaque événement une sphère d'exclusion dont le rayon était égal au rayon de la source, évalué en fonction de la magnitude de l'événement suivant les relations empiriques de Pearson (1982) déjà mentionnées.

La collection de données disponible s'est ainsi trouvée réduite à 14 événements garantis indépendants les uns des autres.

Le résultat de l'inversion (Fig. 17) de ces données est maintenant cohérent avec les données de biréfringence 


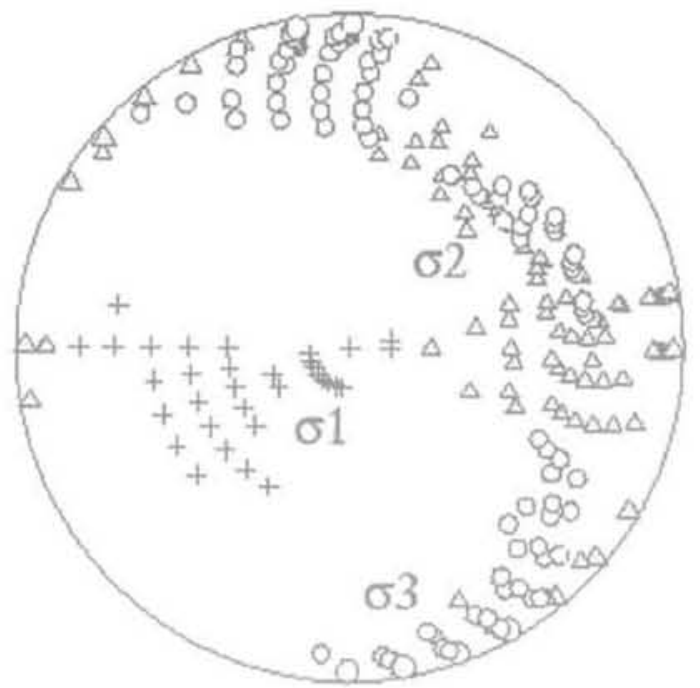

ค16. 16 Directions principales déterminées à partir des mécanismes au foyer observés durant la période juín-juillet 1997. La solution correspond au domaine à $90 \%$ de confiance. ce qui amène à conclure que la faille est perpendiculaire à la direction de contrainte principale minimale. La contrainte maximale est trouvée être la contrainte verticale. A plus grande échelle, les données GPS confirment, effectivement, des mouvements d'extension dans la direction perpendiculaire à la faille.

Ce résultat montre ainsi que cette faille, qui flue à la vitesse d'au moins $2,5 \mathrm{~cm} / \mathrm{an}$, ne supporte aucune composante de cisaillement, ce qui est cohérent avec l'absence d'événement microsismique induit sur la faille lors de l'injection d'eau. Ceci suppose donc un frottement quasi nul, ce qui ne manque pas de poser un certain nombre de questions, notamment sur la rhéologie du matériau de remplissage de la faille.

Ces résultats démontrent la nécessité de pouvoir disposer d'observations à long terme dans les zones de faille, tant du déplacement que du champ de contrainte et du champ de pression interstitielle.

\section{5}

\section{Observations in situ et prévention des risques sismiques}

\section{1}

\section{Observation in situ des processus associés à la déformation crustale, sismique et asismique}

Depuis les premiers modèles de simple dislocation proposés pour les sources sismiques (rappelés en 3.2.2), la compréhension des tremblements de terre a beaucoup progressé grâce à un travail conjoint de spécia-

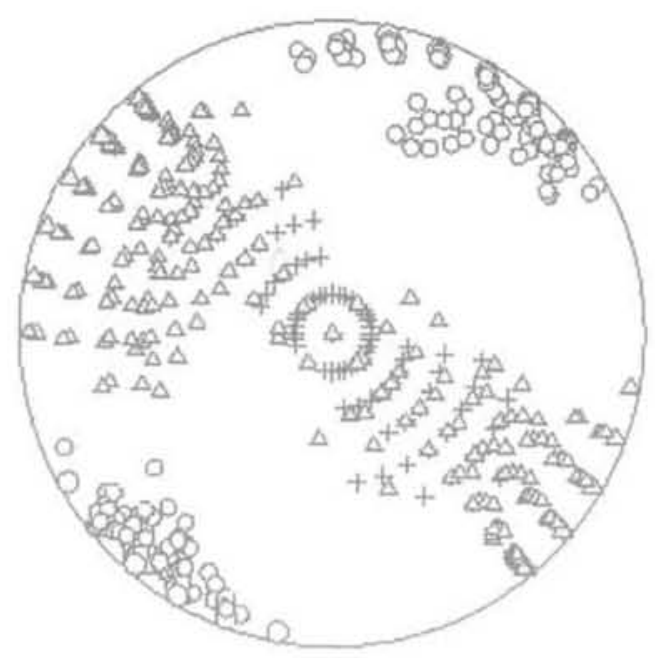

FIG. 17 Orientation des directions principales régionales déterminées à partír des mécanismes au foyer en ne considérant que les événements indépendants (domaine à $90 \%$ de confiance). listes provenant de quasiment toutes les disciplines des sciences de la Terre (sismologie, géomécanique, géodésie, tectonique, mais aussi géochimie, géomagnétisme, hydrogéologie, etc.). Actuellement, de véritables tomographies des ruptures sismiques, réalisées par inversion des sismogrammes de champ proche, décrivent, seconde après seconde, la propagation de la rupture sur la faille lors des grands séismes. A l'autre bout du spectre temporel, les analyses morphotectoniques et les datations sur le terrain révèlent le comportement à long terme d'une faille ou d'un système de faille. Elles décrivent notamment comment les failles intègrent les nombreuses ruptures sismiques dont elles sont le site. Pour des échelles de termps intermédiaires de quelques journées à quelques années, la géodésie par méthode GPS, couplée aux résultats spectaculaires de l'interférométrie SAR, permet de résoudre à l'échelle de plusieurs centaines de kilomètres les déformations intersismiques.

Ces résultats, associés à l'observation de la réponse d'aquifères superficiels à diverses sollicitations sismiques, ont fait prendre conscience de l'importance des interactions fluides-solides. Mais on manque encore de données de terrain, en particulier pour les parties profondes des failles au voisinage de la zone sismogène. De plus, la complexité des phénomènes de couplage hydromécanique reste encore à être démêlée. De même, se fait cruellement sentir le manque de modèle théorique satisfaisant permettant d'expliquer les processus menant aux ruptures instables que sont les séismes.

Une vague d'instrumentation géophysique dense de terrain est ainsi portée par la nécessité de préciser la mécanique crustale sous tous ses aspects, qu'elle concerne les séismes ou les autres processus de déformation plus ou moins lents.

Il s'agit aussi de préciser l'influence des conditions de site sur l'amplification des mouvements sismiques 
ou encore de documenter les conditions requises pour la liquéfaction des sols, en particulier pour les applications à la prévention du risque sismique. En effet, un séisme peut provoquer des dégàts très inégaux sur des sites pourtant proches. Un des enseignements majeurs des séismes survenus ces quinze dernières années (Mexico, 1985; Loma Prieta, 1989; Northridge, 1994 ; Kobe, 1995) est ainsi l'influence des caractéristiques géologiques locales qui peuvent conduire à une altération importante du signal sismique dans le domaine fréquentiel et spectral, désignée sous le nom d'effet de site. De manière générale, la présence d'une couche à faible résistance mécanique se traduit par une amplification de l'amplitude et de la durée du signal sismique. Par ailleurs, pour un sol de type sableux saturé en eau, les vibrations créées par un séisme peuvent conduire à la liquéfaction du sol: le milieu sableux passe d'un état solide à un état liquide sous l'effet de l'augmentation de la pression interstitielle. Les dégâts peuvent alors ètre nombreux. Les catastrophes de Nifigata (Japon. 1964) et de Valdez (1964) où un village entier a été enseveli à la suite d'un phénomène de liquéfaction, ont particulièrement sensibilisé les mécaniciens des sols et ont été à l"origine d'études de laboratoire nombreuses. A ce jour cependant, très rares restent les observations, in situ, de variation du mouvement sismique et/ou de pression de fluide à différentes profondeurs dans un sol liquétié

La compréhension des effets d'un séisme sur les caractéristiques hydromécaniques d'une faille ou d'un massif rocheux est par ailleurs importante pour mieux évaluer la sûreté d'un site de stockage en milieu profond. L'effet principal à considérer concerne la perte éventuelle d'efficacité du confinement apporté par la barrière géologique du fait des variations des caractéristiques hydrauliques du réseau de fracture et des possibilités de perturbation des circulations de fluides dans le massif.

\section{Des interactions fluides-failles actives}

La présence et la circulation de l'eau dans les zones de failles, bien qu'observées depuis longtemps, restent très mal connues et la compréhension de son rōle hydromécanique et chimique dans les processus de tluage et de rupture sismique est encore très incomplète. S'il existe en effet des modẻles numériques et théoriques pour interpréter les couplages poroélas tiques coséismiques, il $n^{\prime} y$ a pour l'instant que des observations indirectes, mal comprises, qui pourraient être liées au processus de rupture dynamique: angles de frottement très faibles de certaines failles, fluages épisodiques des failles et séismes silencieux, propagation de pulses de ruptures étroits sur les failles lors des ruptures sismiques, séismes déclenchés par d'autres à grandes distances, essaims de microséismes, phénomènes précurseurs de certains séismes (variations du niveau ou de la pression des aquifères, de la géochimie des eaux, phénomènes électromagnétiques, etc.).

L'accès à des mesures in situ, au coeur des failles actives, à des profondeurs sismogènes, est donc une étape incontournable pour répondre à ces questions fondamentales sur la mécanique crustale et la génération des séismes. Il s'agit non seulement de caractériser la zone de faille et ses propriêtés par des logs et des carottages, mais aussi d'y établir des mesures conti- nues multiparamètres de pression de pore, de déformation, de géochimie, et de microsismicité.

Dans ou en dehors des zones d'activité sismique, l'évaluation du risque d'instabilité mécanique des pentes continentales, importante pour les implantations pétrolières en offshore protond, est également confrontée à la détermination du rôle joué par les fluides dans le déclenchement de glissements. Les mécanismes qui gènèrent les augmentations et dissipations de la pression des fluides le long des failles, les amplitudes même de ces variations de pression, et les réductions de la contrainte normale effective et du frottement qui en résultent restent mal observés et évalués.

Dans le domaine de l'ingénierie des réservoirs pétroliers, la connaissance de la circulation des fluides dans des roches fracturées et faillées tient aussi un rôle important. La production se fait souvent dans des réservoirs dits fracturés, c'est-à-dire que la plus grande partie des hydrocarbures migre non dans la matrice poreuse mais dans les fractures. La compréhension du comportement hydraulique des grands accidents est donc un enieu de taille. Une faille imperméable peut compartimenter un réservoir, et donc rendre la production du champ difficile, mais aussi empêcher la venue latérale d'eau et donc améliorer la récupération. Les changements des propriétés hydrautiques d'une faille en fonction du champ de pression, qui pour les pétroliers varient plus souvent en fonction de la production et donc de la déplétion des champs que de la sismicité, peuvent avoir des conséquences critiques.

\section{3}

\section{De l'observation sous-marine de circulations de fluides dans des failles actives}

Depuis une dizaine d'années, les géologues marins ont rassemblé de nombreuses observations qui indiquent que les circulations de fluides qui prennent place dans les failles actives des zones de subduction sont un phénomène scientifique de premier ordre (e.g. Suess et al., 1998). La présence de croûtes carbonatées et d'oasis de vie sur le fond marin, de panaches enrichis en méthane dans la colonne d'eau et des mesures directes des flux des fluides expulsés à la surface des sédiments. qui peuvent atteindre des valeurs de plusieurs centaines de litres par $m^{2}$ et par jour, sont des observations aujourd'hui bien connues pour plusieurs marges de subduction (Cascadia, Oregon, Costa Rica, Pérou, Barbade, Aléoutiennes, Japon, Méditerranée....

Les récentes observations de zones de suintements froids sur la marge de Norvège (e,g. McNeill et al., 1998), tout comme celles précédemment décrites par exemple pour la pente du Golfe du Mexique (e.g. Bouma et Roberts, 1990), indiquent que les phénomènes d'expulsion de fluides ne sont pas limités aux seules marges de subduction mais affectent aussi les marges d'extension, dans un contexte de déformation tectonique crustale ou intra-sédimentaire active. Une caractéristique principale de ces sorties de fluides en fond de mer semble être leur nature évolutive dans le temps, avec des variations considérables des vitesses et des flux, qui sont observées sur des périodes de temps aussi courtes que quelques jours, voire quelques heures (Foucher et al., 1992; Tryon et al., 1999).

La mise en place réussie, par une équipe francoaméricaine, d'une station d'observation longue durée 
(sur une période de 18 mois) de la température et de la pression de fluide, dans deux puits ODP forés au front du prisme de la Barbade, a fourni les premières mesures directes de la surpression des fluides dans la faille de décollement d'un prisme d'accrétion, à près de $500 \mathrm{~m}$ sous le fond de la mer (Becker et al., 1997; Foucher et al., 1997), Les valeurs de surpressions mesurées sont modérées, 0,9 à $1,0 \mathrm{MPa}$ dans le puits $949,2,1 \mathrm{MPa}$ dans le puits 948 , soit $1 / 3$ à $1 / 2$ fois les valeurs des surpressions maximales prédites pour un régime de pressions lithostatiques.

Ces résultats démontrent tout l'intérêt qu'il y a à disposer, sur de lonques durées, d'observations de pression et de température en forages recoupant des failles actives. Ils montrent la nécessité que soient développés des sites d'observation continue sur de nombreuses années. Tel est l'un des objectifs du laboratoire géomécanique in situ du Rift de Corinthe actuellement en cours de développement (http://www.corinth-riftlab.org).

\section{REMERCIEMENTS}

Je tiens à remercier ici Jean Sulem, du CERMES, qui est à l'origine de cet article. Je remercie écalement P. Bernard. I. Moretti, F. Cotton et.1.-P. Foucher, qui à un moment ou à un autre, m'ont aidé à préparer le programme d'études en forage dans le rift de Corinthe et à qui jai emprunté certains des éléments de la dernière partie de cet article.
}

\section{Bibliographie}

Baria R., Garnish J., Baumgartner J. Gerard A., Jung R. - Recent developments in the European HDR research programme at Soultz-sous-Forêts (France) n. Proc. World Geothermal Congress, Florence, Italy, vol. 4, 1995 p. $2631-2637$.

Barier E. Huchon P. Aurelio M. - « Philippine fault: a key to Philippine kinema. tics m. Geology, vol. 19, 1991, p. 32-35.

Becker K. Fisher A.T. Davis E.E. - "The CORK experiment in Hole 949 : long term observations of pressure and temperature in the Barbados accretionary prism y. In Shipley T.H., Ogawa T.H. Blum P. Bahr J.M. eds. J. Proc. ODP, Sci. Results, 156: College Station. TX (Ocean Drilling Program), 1997, p. $247-252$

Bouin M.P. Tellez J., Bernard P. - $\alpha$ Seismic anisotropy around the Gulf of Corinth, Greece, deduced from threecomponent seismograms of local earthquakes and its relationship with crustal strain 1. J. Geophys. Res., 101, 1996, p. $5797-5811$

Bouma A.H., Roberts H.H. - $\alpha$ Northerr Gulf of Mexico continental slope v. Geo-Marine Letters, 10, 1990, p. 177 181.

Brudy M. Zoback M. - « Drilling induced tensile wall fractures. Implications for determination of in situ stress orientation and magnitude .. Int. Jour. Rock Mech. Min. Sc., vol. 36, n² 2, 2000 , p. 191-216.

Burridge R., Knopoff L. - $a$ Body force equivalents for seismic dislocations in. Bull. Seism. Soc. Am., vol. 54, 1964, p. $1875-1888$.

Cornet F.H. - Experimental investigation of forced fluid flow through a granitic rock mass $»$. Proceedings of the fourth European Geothermal update (Lou- wrier et al., eds), Kluwer Academic Pub. Dordrecht, 1989, p. 189-204.

Cornet F.H. Valette B - "In situ Stress Determination from Hydraulic Injection Test Data ". Jour Geoph. Res. vol. 89, n B13, 1984, p. 11527-11537.

Cornet F.H., Jones R. - « Field Evidence on the orientation of forced water flow with respect to the regional principal stress directions: panel discussion n. In Nelson and Laubach, ed. Rock Mechanics - Models and Measurements, Bal kema, Rotterdam, 1994, p. 61-71.

Cornet F.H., Yin J. - "Analysis of induced seismicity for stress field determination and pore pressure mapping PAGEOPH, vol. $145, n^{\circ} 3 / 4,1995, p$. $677-700$

Cornet F.H., Helm J., Poitrenaud H., Etchecopar A. - $\alpha$ Seismic and Aseismic Slips Induced by Large Scale Fluid Injections $\gg$, PAGEOPH, vol. $150, n^{\circ} 3 / 4$, 1997 , p. 563-583.

Cornet F.H., Prioul R., Dorbath L., Dorbath C. Herras E.B., Saw V.S. Seastres J.S. Ogena M.S. - "An experiment of mas. sive hyciraulic stimulation in the geothermal field of Tongonnan v. Proceedings of the 19th Annual Geothermal Conf. Manilla Philippines (PNOC-EDC publication), 1998.

Crampin S., Lowell J. - " A decade of shear wave splitting in the Earth crust: what does it mean? what can we make of it? and what to do next? n. Geophys. Jour. Int., vol. 107, 1991, p. 387-407.

Duquesnoy T., Barrier E., Kasser M., Aurelio M., Gaulon R., Punongbayan R., Rangin C. and the french-Philippine Cooperation team - * Detection of creep along the Philippines fault: first results of geodetic measurements on Leyte Island, Central Philippines m. Geophys. Res. Let, vol, 21, 1994, p. 975-978.
Foucher J.P., Henry P., Harmegnies F. a Long-term observations of pressure and temperature in Hole 948D. Barbados accretionary prism p. In Shipley TH. Ogawa TH, Blum P. Bahr, JM. eds. J. Proc. ODP, Sci. Results, vol. 156; College Station, TX (Ocean Drilling Program), 1997, p. 239-245.

Foucher J.P., Henry P., Le Pichon X., Kobayashi K. - "Time-variations of fluid expulsion velocities at the toe of the eastern Nankai accretionary complex 1), Earth and Planetary Science Letters, 109, 1992, p. 373-382

Gamar F., Bernard P., Pham V.N.. Boyer D., Papadimitriou P., Chouliaras G., Charrier A. - "Spatial and temporal variation of seismic and electric anisotropy and its relationship with crustal strain in the Psaromita Peninsula, Gulf of Corinth, Greece n. Submitted to J. Geophys, Res., 2000.

Gaucher E. - Comportement hydroméca. nique d'un massif fracturé : apport de la microsismicité induite - Application au site géothermique du Soultz-sousForêts. Thèse de doctorat de l'université Paris VII. 1998, 245 p.

Gaucher E., Cornet F.H., Bernard P. - Induced seismicity for structure identification and stress field determination $刃$. SPE paper 47 324; Eurock 98, Proc., vol. 1. Soc. Petr. Eng. publ., Richardson, Texas, 1998, p. 545-554.

Gephart J.W., Forsyth D.W. - "An improved method for determining the regional stress tensor using earthquake focal mechanism data: application to San Fernando Earthquake sequencex. J. Geophys, Res., vol. 89, n B11, 1984, p. 9305-9320.

Gérard A., Baumgartner J., Baria R. - « An attempt towards a conceptual Model derived from 1993-1996 Hydraulic ope- 
rations at Soultz m. Proceedings of Nedo International Symposium, vol. 2., 1997, p. 329-341.

Helm J. - The natural seismic hazard and induced seismicity of the European Hot Dry Rock Geothermal Energy Project at Soultz-sous-Forêts. Thèse de doctorat. École et Observatoire de Physique du Globe de Strasbourg, 1996, 197 p.

Jones R.H., Beauce A., Jupe A.. Fabriol H., Dyer B.C. - $\alpha$ Imaging induced microseismicity during the 1993 injection tests at Soultz-sous-Forêts, France n. Proceedings of World Geothermal Congress, Florence, Italy, vol. 4, 1995, p. 2665-2669.

Jutien P., Cornet F.H. - « Stress Determination from Aftershocks of the CaMPania-Lucania Earthquake of November 23, 1980 3. Ann. Geoph., vol. 5b, n 3 . 1987, p. 289-300

Kanamori H., Anderson D.L. - uTheoretical Basis for some Empirical Relations in Seismology y. Buil. Seismol. Soc. Am. vol. $65, n^{\circ} 5,1975$. D. 1075-1095.

Lachenbruch A.H., Sass J.H. - $\alpha$ Heat flow from Cajon Pass, Fault Strength, and Tectonic Implications x. Jour. Geophys. Res., vol. 97, $n^{\circ}$ B4, 1992, p. 4995-5016.

Majer E.L., McEviliy T.V. - « Seismological Investigation at the Geysers geothermal Field n. Science, vol. 274, 1979, p. 82-85.

McGarr A. - $\alpha$ Analysis of States of Stress Between Provinces of Constant Stress 1. Jour. Geophys. Res., vol. 87. $n^{\circ}$ B11, 1982, p. 9279-9288.

McNeill A.E., Salisbury R.S.K., Ostmo S.R, Lien R., Evans D. - «A regional shal- low stratigraphic framework off Mid Norway and observations of deep water special features m. 1998 Offshore Technology Conference abstract volume, 1998, p. 97-109.

Michael A.J. - a Stress rotation during the Coalinga aftershock sequence n. Jour. Geophys. Res., vol. 92, n B8, 1987 . p. $7963-7979$

Mosnier J. - . Détection électrique des fractures naturelles ou artificielles dans un forage 1. Ann. Geophys., vol. 38 1982, p. 537-540.

Nur A., Simmons G. - "Stress induced velocity anisotropy, an experimental study 11. Jour. Geophys. Res, vol. 74, 1969, p. 6667.

Pearson C. - « Parameters and a Magnitude Moment relationship from small Earthquakes Observed during Hydraulic Fracturing Experiments in Crystalline Rocks v. Geophys. Res. Let., vol. 9 , $n^{\circ}$ 4. 1982, p. 404-407.

Prioul R. - Apport de la sismicité induite à l'étude du comportment mécanigue d'un grand décrochement actif: la faille philippine. Thèse de doctorat de I'TP-P. 2000,275 p.

Provost A.S., Houston H. $-\pi$ Orientation of the stress field surrounding the creeping section of the San Andreas Fault: evidence for a narrow mechanically weak fault n. Jour. Geophys. Res., vol. 106, $n^{\circ}$ B6, 2001, p. 11373-11386.

Shapiro S.A., Audigane A., Royer J.J. « Large scale in situ permeability tensors of rocks from induced microseismicity ». Geophys. Jour. Int., vol. 137 1999, p. 207-213.
Shapiro S.A., Audigane A., Royer J.J. "Reply to comment by F.H. Cornt on "Large scale in situ premeability tensor of rocks from induced seismicity ${ }^{\prime}$. Geophys. Jour. Int, vol. 140, 2000. p. $470-473$

Suess F. Bohrmann G von Huene B. Linke P., Wallmann K., Lammers S., H. Sahling - «Fluid venting in the eastern Aleutian subduction zone b. Jour. Geoph. Res, vol. 103, $\mathrm{n}^{\circ}$ B2, 1998, p. $2597-2614$

Talebi S., Cornet F.H. - Analysis of the Microseismicity Induced by a Fluid Injection in a Granitic Rock Mass 1. Geophys, Res, Let., vol, 14, n 3 , 1987 . p. $227-230$

Tryon M.T., Brown K.M., Torres M.E. Trehu A.M., McManus J., Collier R.W. - a Measurements of transience and downward fluid flow near episodic methane gas vents. Hycirate Ridge Cascadia D. Geology, vol. 27, $n^{\circ} 12$, 1999, p. 1075-1078.

Vasseur G.. Etchecopar A. Philip H. (i) Stress state inferred from multiple focal mechanisms v. Ann, Geophys., 1. 1983, p. 291-297.

Yin J., Cornet F.H. - "Integrated stress determination by joint inversion of hydraulic tests and focal mechanisms i. Geophys. Res. Let., vol. 21. $n^{\circ} 24,1994$, p. 2645-2648.

Zoback M.D. Healy J.H. - "In situ Stress Measurements to $3.5 \mathrm{~km}$ Depth in the Cajon Pass Scientific Research Borehole Implication for the Mechanics of Crustal Faulting o, Jour. Geophys, Res., vol. 97, n B4, 1992, p. 5039-5058. 